\title{
Deliquescence, efflorescence, and phase miscibility of mixed particles of ammonium sulfate and isoprene-derived secondary organic material
}

\author{
M. L. Smith ${ }^{1}$, A. K. Bertram ${ }^{2}$, and S. T. Martin ${ }^{1,3}$ \\ ${ }^{1}$ School of Engineering and Applied Sciences, Harvard University, Cambridge, Massachusetts, USA \\ ${ }^{2}$ Department of Chemistry, University of British Columbia, Vancouver, British Columbia, Canada \\ ${ }^{3}$ Department of Earth and Planetary Sciences, Harvard University, Cambridge, Massachusetts, USA \\ Correspondence to: S. T. Martin (scot_martin@ harvard.edu)
}

Received: 20 March 2012 - Published in Atmos. Chem. Phys. Discuss.: 18 April 2012

Revised: 25 August 2012 - Accepted: 6 September 2012 - Published: 23 October 2012

\begin{abstract}
The hygroscopic phase transitions of ammonium sulfate mixed with isoprene-derived secondary organic material were investigated in aerosol experiments. The organic material was produced by isoprene photo-oxidation at $40 \%$ and $60 \%$ relative humidity. The low volatility fraction of the photo-oxidation products condensed onto ammonium sulfate particles. The particle-phase organic material had oxygen-to-carbon ratios of 0.67 to $0.74( \pm 0.2)$ for mass concentrations of 20 to $30 \mu \mathrm{g} \mathrm{m}^{-3}$. The deliquescence, efflorescence, and phase miscibility of the mixed particles were investigated using a dual arm tandem differential mobility analyzer. The isoprene photo-oxidation products induced deviations in behavior relative to pure ammonium sulfate. Compared to an efflorescence relative humidity (ERH) of 30 to $35 \%$ for pure ammonium sulfate, efflorescence was eliminated for aqueous particles having organic volume fractions $\varepsilon$ of 0.6 and greater. Compared to a deliquescence relative humidity (DRH) of $80 \%$ for pure ammonium sulfate, the DRH steadily decreased with increasing $\varepsilon$, approaching a DRH of $40 \%$ for $\varepsilon$ of 0.9 . Parameterizations of the $\operatorname{DRH}(\varepsilon)$ and $\operatorname{ERH}(\varepsilon)$ curves were as follows: $\operatorname{DRH}(\varepsilon)=\Sigma_{i} c_{i, d} \varepsilon^{i}$ valid for $0 \leq \varepsilon \leq 0.86$ and $\operatorname{ERH}(\varepsilon)=$ $\Sigma_{i} c_{i, e} \varepsilon^{i}$ valid for $0 \leq \varepsilon \leq 0.55$ for the coefficients $c_{0, d}=$ $80.67, c_{0, e}=28.35, c_{1, d}=-11.45, c_{1, e}=-13.66, c_{2, d}=0$, $c_{2, e}=0, c_{3, d}=57.99, c_{3, e}=-83.80, c_{4, d}=-106.80$, and $c_{4, e}=0$. The molecular description that is thermodynamically implied by these strongly sloped $\operatorname{DRH}(\varepsilon)$ and $\operatorname{ERH}(\varepsilon)$ curves is that the organic isoprene photo-oxidation products, the inorganic ammonium sulfate, and water form a miscible
\end{abstract}

liquid phase even at low relative humidity. This phase miscibility is in contrast to the liquid-liquid separation that occurs for some other types of secondary organic material. These differences in liquid-liquid separation are consistent with a prediction recently presented in the literature that the bifurcation between liquid-liquid phase separation versus mixing depends on the oxygen-to-carbon ratio of the organic material. The conclusions are that the influence of secondary organic material on the hygroscopic properties of ammonium sulfate varies with organic composition and that the degree of oxygenation of the organic material, which is a measurable characteristic of complex organic materials, is an important variable influencing the hygroscopic properties of mixed organic-inorganic particles.

\section{Introduction}

The water content of atmospheric particles can influence heterogeneous chemistry, cloud activation, and radiative forcing, among other phenomena (Seinfeld and Pandis, 2006). The deliquescence and efflorescence transitions that switch particles between crystalline and aqueous phases, altering the equilibrium particle water content in the process, have been widely studied, typically either for inorganic and organic components separately or for two- or three-component mixtures of them (Tang and Munkelwitz, 1993; Virkkula et al., 1999; Hameri et al., 2000; Martin, 2000; Parsons et al., 2006; Varutbangkul et al., 2006). In the past few years, 
an additional transition of liquid-liquid phase separation between single and multiple liquid phases at a critical relative humidity has been a topic of increasing research interest (Pankow, 2003; Marcolli and Krieger, 2006; Ciobanu et al., 2009; Zuend et al., 2010; Bertram et al., 2011; Smith et al., 2011; Song et al., 2012; You et al., 2012). The separation relative humidity depends both on the identity and the relative concentrations of the organic and inorganic components (Pankow, 2003; Zuend et al., 2010; Bertram et al., 2011). The different morphologies of phase-separated particles, such as coating-core or other structures (Reid et al., 2011), compared to single-phase homogeneous particles can influence the availability of water for heterogeneous chemistry and cloud activation (Ravishankara, 1997), affect particle optical properties (Martin, 2000), and alter the deliquescence and efflorescence phase transition relative humidities (Parsons et al., 2006; Chan and Chan, 2007; Smith et al., 2011).

Laboratory particles of sulfate mixed with secondary organic material represent an important surrogate for a common class of atmospheric particles (Buzorius et al., 2002; Murphy et al., 2006; Pratt and Prather, 2010). These mixed organic-inorganic particles are believed to be produced in the atmosphere by two principal mechanisms. In the first, lowvolatility molecules formed by the atmospheric oxidation of volatile organic compounds (VOCs) condense as secondary organic material (SOM) onto preexisting inorganic particles (Hallquist et al., 2009). In the second, mixed residual particles remain after the evaporation of cloud droplets that contained dissolved inorganic and organic components and in which active scavenging of gas-phase species and subsequent secondary organic chemistry took place during the cloud's lifetime (Ervens et al., 2011). The chemical nature of the inorganic and organic components determines the extent of their interactions and hence the governing organic-inorganicwater phase diagram, including both stable and metastable aspects.

To date, the effects of secondary organic material on the phase transitions of the inorganic components of particles such as ammonium sulfate have been characterized in only a few studies. These studies show that SOM produced by $\alpha$-pinene, limonene, and $\beta$-caryophyllene ozonolysis exerts a minor influence on the deliquescence relative humidity $(\mathrm{DRH})$ and the efflorescence relative humidity (ERH) of the ammonium sulfate component of the mixed particles (Saathoff et al., 2003; Takahama et al., 2007; Bertram et al., 2011; Smith et al., 2011). The implication is a biphasic morphology for the aqueous inorganic and organic components of the particles, at least for the range of water activities associated with DRH and ERH. In the case of $\alpha$-pinene ozonolysis, Smith et al. (2011) showed that DRH and ERH were altered by less than $4 \%$ RH from the values of pure ammonium sulfate for organic volume fractions of up to 0.96 . Although these studies suggest the immiscibility of SOM with an aqueous inorganic phase and thus a minimal influence of
SOM on DRH and ERH of the inorganic phase, the dark ozonolysis products of these precursors represent only a narrow range of possible SOM types. Among other factors, the chemistry and properties of SOM can change with oxidation conditions (e.g., low compared to high $\mathrm{NO}_{\mathrm{x}}$ ) and VOC precursor chemistries (e.g., short chain compared to long chain species). For instance, in contrast with the aforementioned ozonolysis studies, Meyer et al. (2009) provided evidence that SOM produced by $\alpha$-pinene photo-oxidation in the presence of $\mathrm{NO}_{\mathrm{x}}$ can decrease the DRH of ammonium sulfate by at least $5 \%$, though the exact decrease was not quantified. Bertram et al. (2011) likewise presented an initial data set for the effects of SOM produced by isoprene photo-oxidation on the phase transitions of ammonium sulfate, showing a decrease in DRH for organic volume fractions from 0 to 0.8 and a decrease in ERH for organic volume fractions from 0 to 0.6. Compared to the other SOM types studied, this isoprenederived SOM appeared to have a markedly different effect on the phase transitions of ammonium sulfate.

In the present study, a comprehensive data set for the effects of SOM produced by isoprene photo-oxidation on the phase transitions of ammonium sulfate was collected for a broad range of organic volume fractions from 0.0 up to 0.86 . The data set is more comprehensive and the experimental protocols and analysis are improved compared to our earlier treatment in Bertram et al. (2011). These improvements allow a better assessment of phase miscibility, a more accurate parameterization of $\operatorname{DRH}(\varepsilon)$ and $\operatorname{ERH}(\varepsilon)$, and a new account of partial dissolution of ammonium sulfate for $\mathrm{RH}<\mathrm{DRH}$ (i.e., initial deliquescence) for these mixed particles. The influence of different conditions of SOM production, including in the presence of aqueous compared to solid sulfate particles at relative humidities of 40 and $60 \%$, is also explored.

\section{Experimental}

\subsection{Particle generation}

Particles of ammonium sulfate having isoprene-derived SOM coatings were produced using the Harvard Environmental Chamber (HEC) (Shilling et al., 2008; King et al., 2009). The chamber was operated as a continuously mixed flow reactor (CMFR), with balanced inflows and outflows and a mean reactor residence time of $3.3 \mathrm{~h}$. Ammonium sulfate particles (Fluka TraceSELECT, > $99.9999 \%$ purity), gaseous isoprene (200 ppb; Scott Specialty Gases, 50 ppm in nitrogen), and gaseous hydrogen peroxide (ca. $10 \mathrm{ppm}$ ) were continuously injected. The hydrogen peroxide was evaporated from a solution (Sigma-Aldrich, $30 \mathrm{wt} \%$ solution) in a warmed bulb. The sulfate particles, produced by atomization from an aqueous solution, were equilibrated to less than $30 \%$ relative humidity $(\mathrm{RH})$, unless stated otherwise, by passing the aerosol through a diffusion dryer. Aqueous ammonium sulfate particles in pure form effloresce between $30 \%$ and 
$35 \%$ RH to form solidified particles (Martin, 2000). Prior to entering the CMFR, the sulfate particles were classified using a differential mobility analyzer (DMA) that was set to pass an electric-field mobility diameter $d_{\mathrm{m}+1}^{\text {seed }}$ (i.e., +1 chargeequivalent) of 68 to $72 \mathrm{~nm}$ (Knutson and Whitby, 1975). The resolution of the DMA for the operating conditions corresponded to singly charged particle diameters as small as $61 \pm 2 \mathrm{~nm}$ and as large as $83 \pm 2 \mathrm{~nm}$. Doubly charged sulfate particles, characterized by a mode diameter between 99 and $103 \mathrm{~nm}$ and a total diameter range of $90 \pm 2$ to $120 \pm 2 \mathrm{~nm}$, were also transmitted through the DMA and entered the CMFR. The +1 and +2 solid particles were present in the CMFR inflow in an approximate ratio of 5:1. The seed number-diameter distribution was measured using a TSI Scanning Mobility Particle Sizer (SMPS) (Fig. S1) (Wang and Flagan, 1990).

Within the CMFR, photolysis of $\mathrm{H}_{2} \mathrm{O}_{2}$ by ultraviolet light produced $\mathrm{OH}$ radicals that initiated photo-oxidation of the isoprene (Kroll et al., 2006). The $\mathrm{OH}$ concentration was estimated as approximately $2 \times 10^{6} \mathrm{~cm}^{-3}$ based on the steady-state concentration of isoprene in the outflow from the CMFR. Except when noted otherwise, experiments were conducted at $40 \% \mathrm{RH}$. Low-volatility gas-phase products of the isoprene oxidation condensed onto the surfaces of the particles present in the CMFR, increasing particle diameter. The particles had a range of individual residence times because Poisson statistics governed particle loss in the CMFR, both to the walls as well as in the outflow (Kuwata and Martin, 2012). At steady state, the number-diameter distribution of the particles in the CMFR outflow was controlled by three main factors: (1) the number concentration and diameters of the +1 and +2 particles in the CMFR inflow, (2) the rate of condensable product generation by isoprene photo-oxidation, and (3) the particle mean residence time in the CMFR. For the conditions of the experiments, the mode of the particle population in the CMFR outflow was between 151 and $172 \mathrm{~nm}$, and the lower and upper quartile diameters were between 95 and 103 and 242 to $257 \mathrm{~nm}$, respectively, as measured by the SMPS at $25 \%$ RH (Fig. S1). The total number concentration in the CMFR outflow was $7 \pm 1 \times 10^{3} \mathrm{~cm}^{-3}$.

For a single seed diameter (i.e., either +1 or +2 seed particles), larger particles in the CMFR outflow corresponded to longer residence times and thus higher organic volume fractions. The particle population in the CMFR outflow consisted of two sub-populations: for any outflow diameter equal to or greater than $90 \mathrm{~nm}$ (i.e., the lower limit of +2 seed particles) there was one sub-population of organic volume fractions associated with the +1 seed particles and another subpopulation associated with the +2 seed particles.

The steady-state organic particle mass concentration, which is a measure of the rate of condensable product generation, ranged from 20 to $30 \mu \mathrm{g} \mathrm{m}^{-3}$. The concentration was measured using an Aerodyne Aerosol Mass Spectrometer (AMS) (DeCarlo et al., 2006; Aiken et al., 2007; Chen et al.,
2011). The AMS also provided information on the oxygento-carbon elemental ratio of the particle-phase organic material, which varied from 0.67 to 0.74 for the experiments described herein. The uncertainty of the oxygen-to-carbon elemental ratio by this method was \pm 0.2 (Aiken et al., 2007).

\subsection{Particle hygroscopic analysis}

The hygroscopic properties of the particle population in the CMFR outflow were analyzed using a dual-arm hygroscopic tandem differential mobility analyzer (TDMA) (Martin et al., 2008; Rosenoern et al., 2009; Smith et al., 2011). Figure 1 shows schematically the flow path of the aerosol from the generation system through the TDMA components, along with the typical RH of each component, to be discussed below. Two different types of experiments, described as transmission ratio and number-diameter distribution measurements, were carried out.

\subsubsection{Transmission ratio experiments}

The transmission ratio experiments directly measured the fraction of the particles in the test population that underwent irreversible diameter changes as a result of RH cycling, such as those associated with deliquescence and efflorescence phase transitions (Rosenoern et al., 2009; Smith et al., 2011). In overview, the measurement consisted of selecting a specific particle diameter with a differential mobility analyzer (DMA), perturbing the RH of the aerosol, and measuring the number concentration remaining at the original diameter (i.e., using a second DMA and a condensation particle counter (CPC)). A drop in number concentration at the second compared to the first DMA indicated that particles had changed diameter in response to the RH perturbation.

More specifically, the measurement protocols were as follows. The incoming flow of the TDMA was conditioned to $\mathrm{RH}^{\text {mono }}$ by use of a Nafion tube (Perma Pure, PD50T12SS). A first differential mobility analyzer (DMA ${ }^{\text {mono }}$; TSI, $3081 ; 9.6: 1$ sheath:sample flow ratio; $25^{\circ} \mathrm{C}$ ) selected a fraction of the particle population having an electric-field mobility diameter $d_{\mathrm{m},+1}^{\text {mono }}$. The sheath flow RH was adjusted to $\mathrm{RH}^{\mathrm{mono}}$ prior to entering the DMA. Following DMA ${ }^{\text {mono }}$, the flow was split and directed into dual arms, labeled $\alpha$ and $\beta$. Each of these arms consisted of the following components in series: a Nafion tube (Perma Pure, MD 110-96SS) set to a value $\mathrm{RH}_{1, \alpha / \beta}$, a Nafion tube set to $\mathrm{RH}^{\text {mono }}$, a differential mobility analyzer $\mathrm{DMA}_{\alpha / \beta}^{\text {filter }}$ (TSI, 3081; ca. 9.6:1 sheath:sample flow ratio; $25^{\circ} \mathrm{C}$ ) set to pass the same electricfield mobility diameter as $d_{\mathrm{m},+1}^{\text {mono }}$ and maintained at $\mathrm{RH}^{\mathrm{mono}}$, and a condensation particle counter (CPC; TSI, 3010) to measure the number concentration $N_{\alpha / \beta}^{\text {filter }}$ of particles passing $\mathrm{DMA}_{\alpha / \beta}^{\text {filter }}$. The experiment consisted of measuring the transmission ratio $\phi$ (calculated as $\phi=N_{\beta}^{\text {filter }} / N_{\alpha}^{\text {filter}}$ ) while holding $\mathrm{RH}_{1, \alpha}^{\text {nafion }}$ fixed to the same value as $\mathrm{RH}^{\text {mono }}$ and 

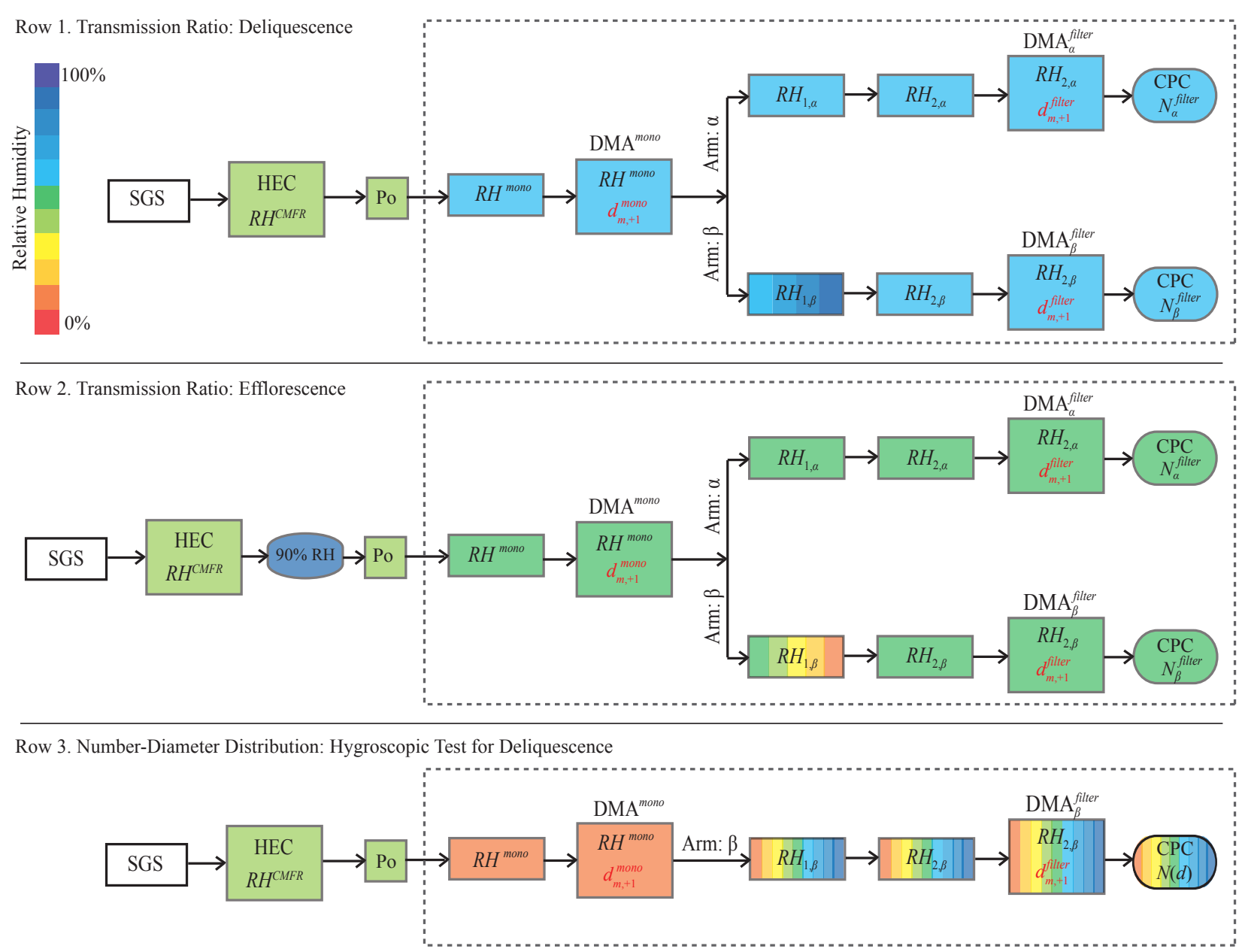

Row 4. Number-Diameter Distribution: Hygroscopic Control for Forced Deliquescence

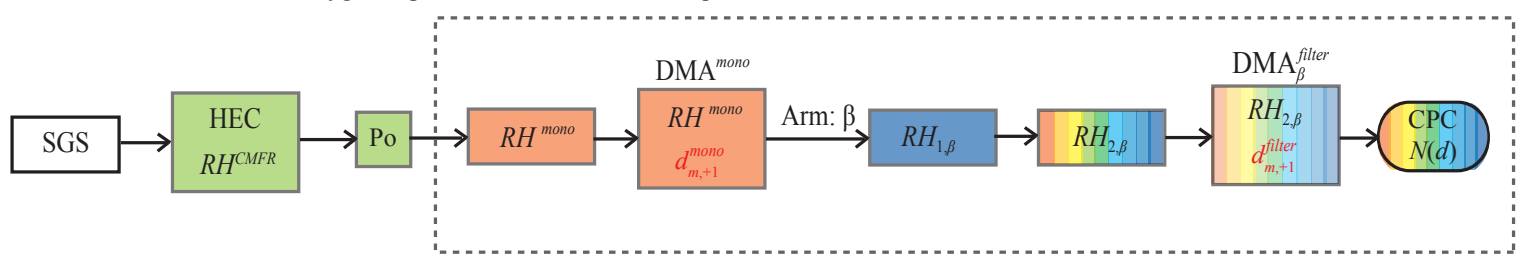

Fig. 1. Schematic diagram of the experimental setup for the transmission ratio and number-diameter distribution measurements. Labeled components include the sulfate seed particle generation system (SGS), the Harvard Environmental Chamber (HEC), a polonium-210 charger (Po), relative humidity (RH) adjustments in Nafion tubes, differential mobility analyzers (DMA), and condensation particle counters (CPC). The background color shading of each component represents the local relative humidity (see color bar). Rainbow shading within a single component indicates that the relative humidity is scanned during the measurements. The gray dashed boxes enclose the components that comprise the TDMA.

scanning $\mathrm{RH}_{1, \beta}^{\text {nafion }}$. The purpose of the $\alpha$-arm was to provide a control for fluctuations in the number concentration of $d_{\mathrm{m},+1}^{\mathrm{mono}}$ in the CMFR outflow by counting how many particles passed through the instrument in the absence of $\mathrm{RH}$ perturbations.
For experiments designed to identify deliquescence transitions (Fig. 1, row 1; Fig. 2a), $\mathrm{RH}_{1, \beta}^{\text {nafion }}$ was scanned upward from $60 \%$ to $90 \%$ during the course of an experiment while $\mathrm{RH}^{\mathrm{mono}}$ was maintained at $60 \%$. In this way, the particles in the $\beta$ arm underwent an RH history described by the 
following:

$60 \% \rightarrow d_{\mathrm{m},+1}^{\mathrm{mono}} \rightarrow 60 \%+\delta \rightarrow 60 \%$

for $0<\delta<30 \%$. For experiments designed to identify efflorescence transitions (Fig. 1, row 2; Fig. 2c), the particle population in the CMFR outflow was deliquesced at $90 \% \mathrm{RH}$ prior to entering the TDMA instrument. The transmission ratio was recorded for a downward scan of $\mathrm{RH}_{1, \beta}^{\text {nafion }}$ from $50 \%$ to $15 \%$ while $\mathrm{RH}^{\text {mono }}$ was maintained at $50 \%$. The $\mathrm{RH}$ history of the particles was thus as follows:

$90 \% \rightarrow 50 \% \rightarrow d_{\mathrm{m},+1}^{\mathrm{mono}} \rightarrow 50 \%-\delta \rightarrow 50 \%$

for $0<\delta<35 \%$. The lower limit of $15 \%$ emerged as a practical matter based on the time constant for Nafion material to equilibrate and transfer local relative humidity, which was longer at progressively lower RH, compared to the time constant desired for an experimental scan of approximately $1 \% \mathrm{RH} \mathrm{min}^{-1}$. A series of these deliquescence and efflorescence experiments was carried out in which stepwise changes were made to $d_{\mathrm{m},+1}^{\text {mono }}$ between experiments. The range of $d_{\mathrm{m},+1}^{\text {mono }}$ values studied was between 75 and $120 \mathrm{~nm}$, implying an associated range of low to high organic volume fractions (specified in greater detail further below). The RH histories of Eqs. (1) and (2) are summarized in Table S1.

For the conducted experiments, the transmission ratio $\phi$ responded to the fraction of particles in the population for which an irreversible change in diameter occurred when exposed to the cycle of $\mathrm{RH}^{\text {mono }} \rightarrow \mathrm{RH}_{1, \beta}^{\text {nafion }} \rightarrow \mathrm{RH}^{\text {mono }}$ (Rosenoern et al., 2009; Smith et al., 2011). A direct interpretation of $\phi$ as the actual fraction of particles for which the diameters changed irreversibly (i.e., a linear response factor) was possible provided that the shift from one branch to the other of the hysteresis curve changed the particle diameter sufficiently to ensure negligible particle transmission through the DMA $^{\text {mono_to-DMA }}{ }_{\beta}^{\text {filter }}$ sequence. For the employed operating conditions, the minimum growth factor $g$ required for full separation was 1.12 (cf. Eq. (2) of Rosenoern et al. (2009) and Figs. 5 and 6 of Smith et al., 2011).

\subsubsection{Number-diameter distribution experiments}

The transmission ratio experiments described in Sect. 2.2.1 were restricted by the experimental protocols to particles that fully deliquesced above $60 \% \mathrm{RH}$ in the deliquescence mode experiments and particles that had efflorescence points greater than $15 \% \mathrm{RH}$ in the efflorescence mode experiments. The number-diameter distribution experiments, as described below, pushed these limits to as low as $48 \%$ RH for deliquescence measurements and as low as $7 \% \mathrm{RH}$ for efflorescence measurements. The experimental design and data analysis, however, were more complicated.

The apparatus, when used in number-diameter distribution mode, is similar to a Hygroscopic Tandem Differential Mobility Analyzer (HTDMA) (Duplissy et al., 2009). In overview, the protocol is to size-select quasi-monodisperse particles under dry conditions, expose this aerosol to an elevated $\mathrm{RH}$, and measure the associated number-diameter distribution. Differences between the grown and initial distributions indicate water uptake (or loss).

A detailed description of the protocol is as follows: A number-diameter distribution $N(d)$ was collected in a single arm for some fixed value of $d_{\mathrm{m},+1}^{\mathrm{mono}}$ and a constant $\mathrm{RH}^{\mathrm{mono}}$ value of $7 \%$, meaning particles classified by $\mathrm{DMA}^{\text {mono }}$ had minimal water content (Fig. 1, rows 3 and 4). To acquire $N(d)$, we recorded the number concentration $N^{\text {filter }}$ as $d_{\mathrm{m},+1}^{\text {filter }}$ was scanned. During a single scan of $d_{\mathrm{m},+1}^{\text {filter }}$, the values $\mathrm{RH}_{1}^{\text {nafion }}$ and $\mathrm{RH}_{2}^{\text {nafion }}$ of the two Nafion tubes remained constant. Between scans, these RH values were changed, and a data set $N(d, \mathrm{RH})$ was thereby obtained for one $d_{\mathrm{m},+1}^{\mathrm{mono}}$. The $\mathrm{RH}$ histories that were employed for $\mathrm{RH}_{1}^{\text {nafion }}$ and $\mathrm{RH}_{2}^{\text {nafion }}$ are presented in Sect. 3.2 because of their close coupling to the data interpretation. The value of $d_{\mathrm{m},+1}^{\mathrm{mono}}$, fixed during the acquisition of a single data set $N(d, \mathrm{RH})$, was adjusted between experiments to a small number of selected values (cf. Table S1) ranging from 75 to $190 \mathrm{~nm}$ so that data sets of $N(d, \mathrm{RH})$ for different regions of organic volume fraction were obtained. The data sets $N(d, \mathrm{RH})$ are reported as recorded without any corrections for the presence of multiply charged particles (Wang and Flagan, 1990). Instead, a forward-model was used that includes a treatment of the particle sub-populations in the outflow from the CMFR (cf. Sect. 3.1).

In comparison to the transmission ratio experiments, the number-diameter distribution measurements could probe efflorescence to lower $\mathrm{RH}$. In these experiments, $\mathrm{RH}^{\mathrm{mono}}$ was set to a static value of $7 \% \mathrm{RH}$, which could be achieved after sufficient equilibration time of the Nafion material. Furthermore, measurements of deliquescence were also extended to lower RH values by detailed interpretation of the data that considered the several particle types present in the population and the resulting contributions to $N(d)$ (cf. Sect. 3.2) in the number-diameter distribution experiments. Compared to the theoretical lower limit of $40 \% \mathrm{RH}$ that is implied by the experimental $\mathrm{RH}$ inside the CMFR, there was a practical restriction to $48 \% \mathrm{RH}$ in these deliquescence experiments because of a coupling of factors, including the accuracy of sizing resolution by the instrumentation and the decrease in diameter hysteresis for increasing $\varepsilon$.

\subsubsection{Other number-diameter distribution experiments}

To extend the range of efflorescence measurements to $7 \%<\mathrm{RH}<15 \%$, we collected a few number-diameter distributions following the protocol of the transmission ratio efflorescence experiments. Aqueous particles of $d_{\mathrm{m},+1}^{\mathrm{mono}}$ were selected at $50 \% \mathrm{RH}$, and the $\mathrm{RH}$ cycling in the TDMA was identical to that defined by Eq. (2). The measured quantity, however, was $N(d, 50 \%)$ instead of $\phi$. The range 
$7 \%<\mathrm{RH}_{1, \beta}^{\text {nafion }}<15 \%$ was obtained by using $3 \mathrm{~h}$ equilibration times for each step of the Nafion conditioner. This protocol was also used in several control experiments to probe ERH between $15 \%$ and $50 \%$ to test for and ultimately rule out any possible bias introduced by scan time or alternate approaches to data analysis.

\section{Results and discussion}

\subsection{Particle population and sub-populations}

The particle population in the outflow of the CMFR is statistically described by first and third quartile diameters of approximately 100 and $250 \mathrm{~nm}$, respectively, at $25 \% \mathrm{RH}$. Individual particles are composed of sulfate and organic components. The sulfate component represents a mass-equivalent diameter of either $61-83 \mathrm{~nm}$ or $90-120 \mathrm{~nm}$, corresponding to initial seed particles of +1 or +2 charge. The water content at $25 \% \mathrm{RH}$ is small by volume compared to the sulfate and organic components: the mixed particles on the lower branch of the hysteresis curve had a hygroscopic diameter growth factor of less than 1.02 at $25 \% \mathrm{RH}$.

The particle population exiting the CMFR is re-charged by a radiation source as it enters the TDMA (Fig. 1). Correspondingly, by number concentration there are four signif-

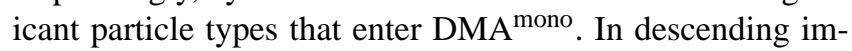
portance, these sub-populations include: (A) singly charged particles having 61-83 nm sulfate seeds, (B) doubly charged particles having $61-83 \mathrm{~nm}$ seeds, (C) singly charged particles having 90-120 nm seeds, and (D) doubly charged particles having a $90-120 \mathrm{~nm}$ seeds. The relative concentration of each particle type at the setpoint value $d_{\mathrm{m},+1}^{\mathrm{mono}}$ determines the composition of the particle population that exits DMA ${ }^{\text {mono }}$. Particles of type $\mathrm{C}$ have non-zero concentrations after $\mathrm{DMA}^{\text {mono }}$ only for $d_{\mathrm{m},+1}^{\mathrm{mono}}>90 \mathrm{~nm}$.

The sub-population probability density functions $p(\varepsilon ; T)$ of organic volume fraction $\varepsilon$ for the four particle types $T$ (i.e., A, B, C, and D) in the outflow of $\mathrm{DMA}^{\text {mono }}$ are obtained by use of a model. Model inputs include the measured number-diameter distributions of the seed particles in the CMFR inflow and of the particle population in the CMFR outflow. Further information about the model is presented in the Appendix. Examples of the cumulative distribution function $P(\varepsilon)$ of the entire particle population are plotted in panels $\mathrm{b}$ and $\mathrm{d}$ of Fig. 2. Examples of the probability density functions $p(\varepsilon ; T)$ of the four different sub-populations are represented by the colored lines in panels $b$ and $d$ of Fig. 3 .

\subsection{Deliquescence and efflorescence}

\subsubsection{Transmission ratios}

Solid ammonium sulfate particles in pure form deliquesce at $80 \% \mathrm{RH}$ to form purely liquid particles (Martin, 2000). When mixed with other components, ammonium sulfate and those components can form a mixed aqueous phase at a relative humidity less than $80 \%$, often called the initial deliquescence RH or eutonic RH. The mixed phase persists in equilibrium with pure ammonium sulfate (alternatively, in equilibrium with the other components depending on the relative initial mass of each) across a finite RH range prior to full dissolution into a mixed phase, often referred to as final deliquescence. Particles in the RH range of partial dissolution lie on the lower side of the hysteresis curve because the residual pure phase solid material typically grows and dissolves without an activation barrier, leading to reversible changes in particle diameter with RH cycling. By comparison, full dissolution at final deliquescence shifts a particle from the lower to the upper side of the hysteresis curve. Irreversible changes in particle diameter are associated with $\mathrm{RH}$ cycles that pass through full dissolution. A pictorial representation of partial and full dissolution is illustrated in Fig. S3.

The organic volume fraction at which final deliquescence occurs for a fixed RH value is denoted by $\varepsilon_{\mathrm{D}}(\mathrm{RH})$. The Gibbs-Duhem thermodynamic relationship describes the curve $\varepsilon_{\mathrm{D}}(\mathrm{RH})$ for decreasing RH (Berry et al., 2000). The particles in the CMFR outflow having $\varepsilon<\varepsilon_{\mathrm{D}}(40 \%)$ are on the lower side of the hysteresis branch because the seed particles in the CMFR inflow are injected as solid sulfate particles at $40 \% \mathrm{RH}$. This sub-population having $\varepsilon<\varepsilon_{\mathrm{D}}(40 \%)$ can be probed for deliquescence transitions by cycling RH.

An example of a data set of transmission ratios used to identify deliquescence transitions is plotted in Fig. 2a. The drop from unity of the transmission ratio $\phi$ for increasing $\mathrm{RH}$ is proportional to the fraction of the particle population that has undergone irreversible deliquescence. The curve $\phi(\mathrm{RH})$ of Fig. 2a can then be interpreted as $\mathrm{DRH}(\varepsilon)$ by pointto-point matching of $\phi(\mathrm{RH})$ to the cumulative distribution function $P(\varepsilon)$ of organic volume fraction of Fig. $2 b$. The data of Fig. 2a, plotted for $d_{\mathrm{m},+1}^{\mathrm{mono}}=90 \mathrm{~nm}$, show that the transmission ratio drops from unity to 0.19 as $\mathrm{RH}_{1, \beta}^{\text {nafion }}$ is scanned from $73 \%$ to $79 \%$. The interpretation is that particles deliquescing at the highest $\mathrm{RH}$ values, corresponding to the green point of Fig. 2a, have the lowest organic volume fraction, corresponding to the green point of Fig. $2 b$. The pink point in Fig. $2 \mathrm{a}$ and $\mathrm{b}$ shows that particles of $\varepsilon=0.58$ have a deliquescence point of $73 \% \mathrm{RH}$. This result is written in shorthand as $\varepsilon_{\mathrm{D}}(73 \%)=0.58$. The conclusion from Fig. 2a and $\mathrm{b}$ is that DRH drops from $80 \%$ for pure ammonium sulfate to $73 \%$ for $\varepsilon$ of 0.58 . The set of points $\varepsilon_{\mathrm{D}}(\mathrm{RH})$ for $73 \%<\mathrm{RH}<80 \%$ of Fig. $2 \mathrm{a}$ and $\mathrm{b}$ represent a curve of $\varepsilon_{\mathrm{D}}(\mathrm{RH})$ across the domain $0<\varepsilon_{\mathrm{D}}<0.58$. Swapping the dependent variable $\varepsilon$ and the independent variable RH, the line $\operatorname{DRH}(\varepsilon)$ is constructed and appears in Fig. 4. Examples of other data sets are shown in Fig. S4.

The pink point of Fig. 2a and $\mathrm{b}$ represents the beginning of a gap in the continuity of $\varepsilon$, separating particles of types $A$ and $D$ from those of type $B$. As a result, there are very few particles in the range $0.6<\varepsilon<0.7$. Fig. $2 \mathrm{a}$ and $\mathrm{b}$ show that 


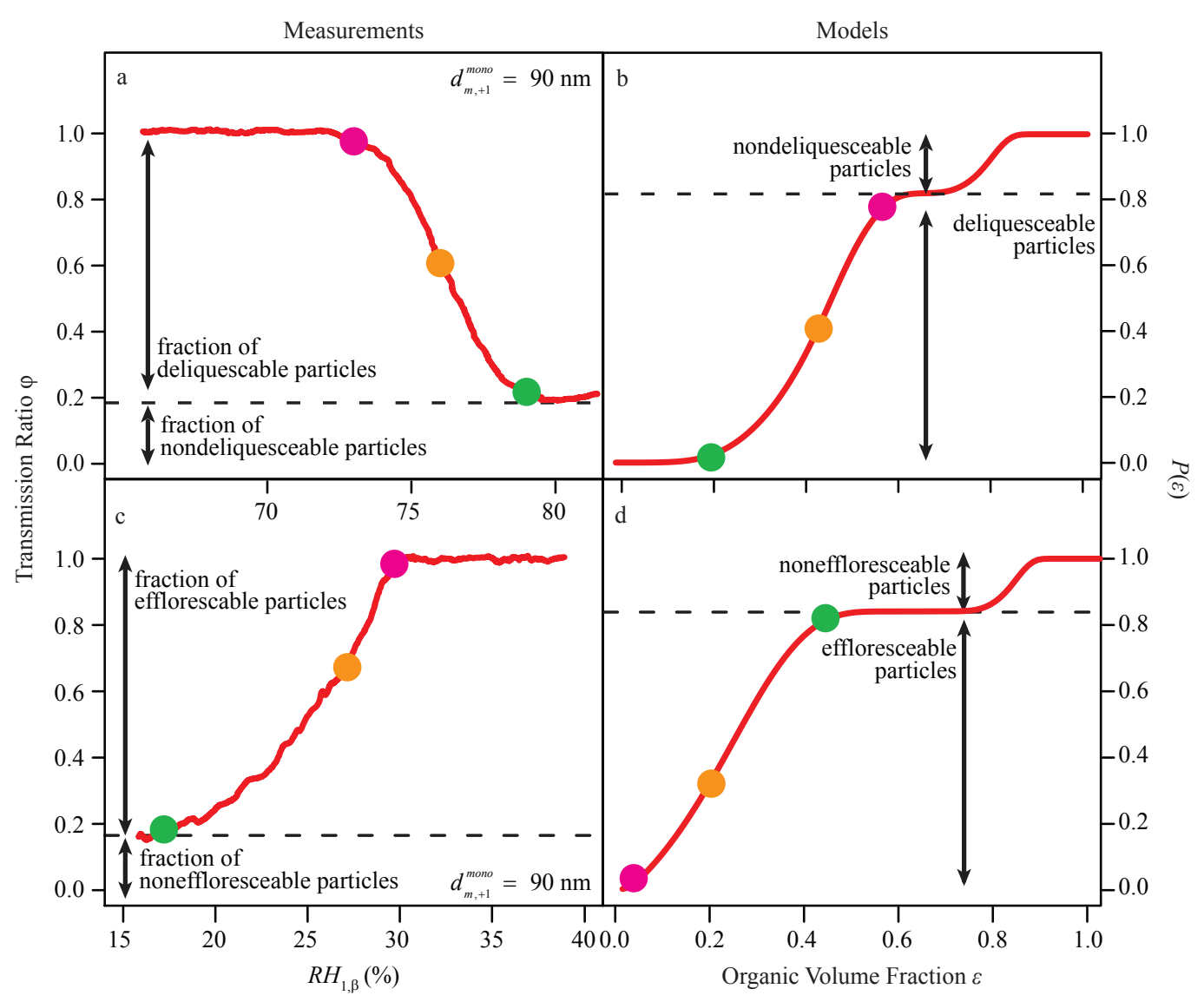

Fig. 2. Examples of data sets and associated analysis for transmission ratio experiments. (a) Transmission ratio $\phi$ of the particle population as $\mathrm{RH}_{1, \beta}$ is scanned upward. These experiments are designed to identify deliquescence transitions. The ratio drops from unity as the particles deliquesce. (b) Cumulative distribution function of organic volume fraction $\varepsilon$ for this particle population. The colored circles of (a) and (b) show points of correspondence between the $\phi(\mathrm{RH})$ and $P(\varepsilon)$ curves (i.e., the green circle shows that the highest DRH value matches with the lowest value of $\varepsilon$ ). The corresponding points of each curve are the basis of the $\operatorname{DRH}(\varepsilon)$ curves shown in Fig. 4. Panels (c) and (d) are as described for (a) and (b) with the difference that $\mathrm{RH}_{1, \beta}$ is scanned downward in experiments designed to identify efflorescence transitions. The corresponding data points of (c) and (d) are the basis of the $\operatorname{ERH}(\varepsilon)$ curves shown in Fig. 4.

the particles in the range of $\varepsilon=0.7$ to 0.85 do not show an irreversible change in diameter upon RH cycling. The implication, given that the RH cycle is of $60 \% \rightarrow d_{\mathrm{m},+1}^{\mathrm{mono}} \rightarrow$ $60 \%+\delta \rightarrow 60 \%$ for $0<\delta<30 \%$, is that the mixed particles of ammonium sulfate and isoprene-derived SOM fully deliquesce at $60 \% \mathrm{RH}$ for some value of $\varepsilon$ that lies between 0.6 and 0.85 , meaning $0.6<\varepsilon_{\mathrm{D}}(60 \%)<0.85$.

An example of a data set of transmission ratios used to identify efflorescence transitions is plotted in Fig. 2c. The cumulative distribution function $P(\varepsilon)$ of organic volume fraction for the particle population is plotted in Fig. 2d. In this population, all particles begin as aqueous because of exposure to $90 \% \mathrm{RH}$ prior to entering the TDMA. The data of Fig. 2c show that $82 \%$ of the particles effloresce between $15 \%$ and $30 \% \mathrm{RH}$ and that no particles effloresce above $30 \% \mathrm{RH}$. The particles efflorescing at the highest RH values, corresponding to the pink point of Fig. 2c, have the lowest organic volume fractions, corresponding to the pink point of
Fig. 2d. The green point in Fig. $2 \mathrm{c}$ and d shows that particles of $\varepsilon=0.43$ effloresce at $15 \% \mathrm{RH}$. This result is written in shorthand as $\varepsilon_{\mathrm{E}}(15 \%)=0.43$ and means that for an efflorescence relative humidity of $15 \%$ the organic volume fraction is 0.43 . As for Fig. 2a and b, this analysis is extended to obtain a curve $\varepsilon_{\mathrm{E}}(\mathrm{RH})$, and this curve is represented in Fig. 4.

Figure $2 \mathrm{~d}$ shows that there are very few particles in the population having $0.43<\varepsilon<0.8$. For $\varepsilon>0.8$, particles do not show an irreversible change in diameter upon RH cycling (Fig. 2c). Given that the RH cycle is $90 \% \rightarrow 50 \% \rightarrow$ $d_{\mathrm{m},+1}^{\mathrm{mono}} \rightarrow 50 \%-\delta \rightarrow 50 \%$ for $0<\delta<35 \%$, possible explanations are (a) that these mixed particles of ammonium sulfate and isoprene-derived SOM are already fully effloresced at $50 \% \mathrm{RH}$, (b) that ERH is below $15 \%$ for particles of $\varepsilon>0.8$, or (c) that the solubility of the ammonium sulfate within the organic material exceeds $(1-\varepsilon)$ even below $15 \% \mathrm{RH}$. In other words, ammonium sulfate is not supersaturated even below $15 \% \mathrm{RH}$ for aqueous solutions of 


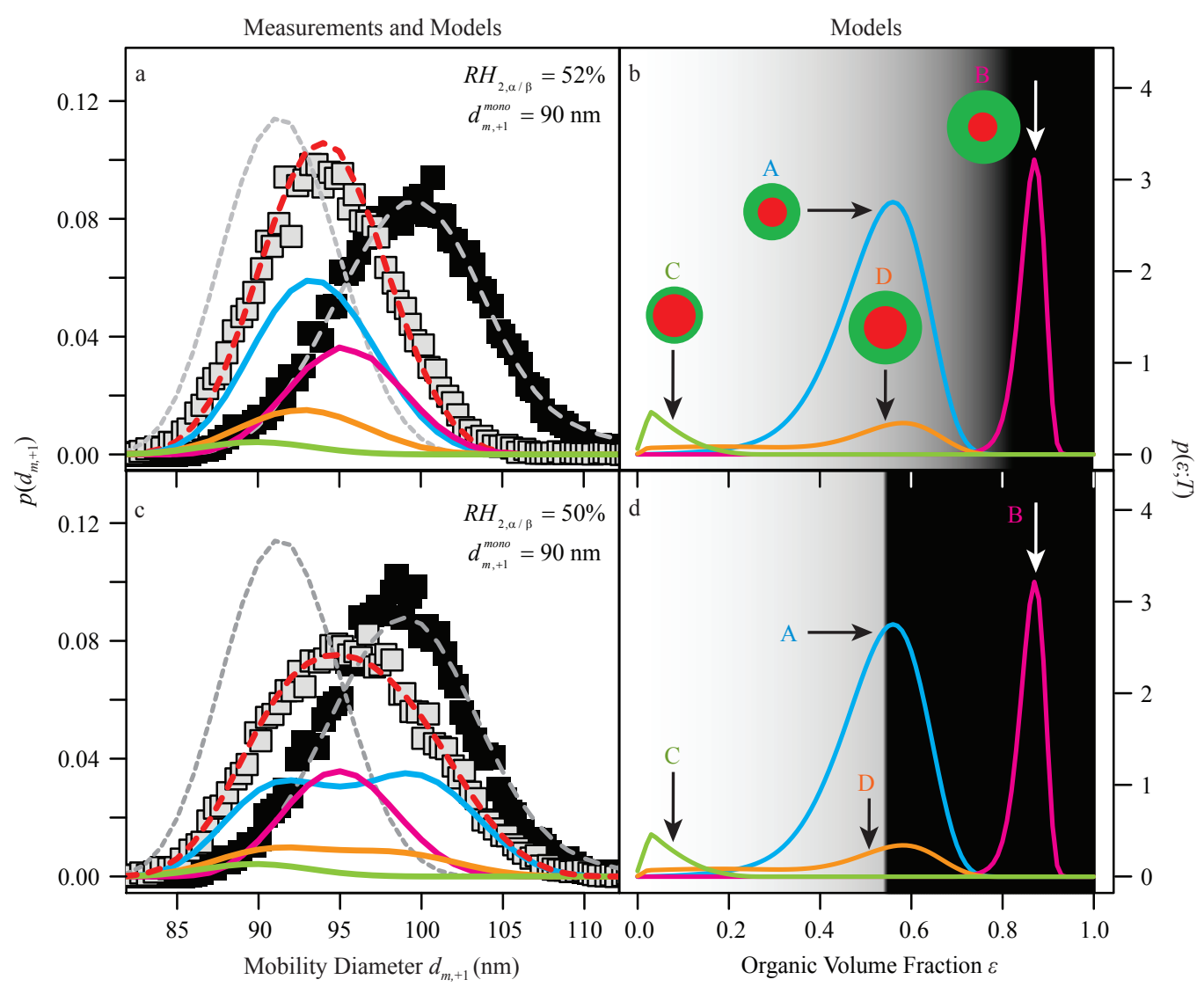

Fig. 3. Examples of data sets and associated analysis for number-diameter distribution experiments. The black squares of (a) correspond to a population in which the ammonium sulfate components of all particles are fully deliquesced because of exposure to $90 \% \mathrm{RH}$ and the absence of efflorescence at $52 \% \mathrm{RH}$. The gray squares correspond to a population in which the ammonium sulfate components of some fraction of the particles are fully deliquesced (i.e., those particles of $\varepsilon>\varepsilon_{\mathrm{D}}(52 \%)$ ). That fraction, as well as $\varepsilon_{\mathrm{D}}(52 \%)$, are unknown prior to measurement and analysis. The solid colored lines represent model results for the different particle sub-populations represented in (b). The dashed lines represent model results for the entire particle population, with the red dashed lines as the sum of the other four colored lines, the short-dashed gray lines as a population in which the ammonium sulfate components of the particles are crystalline, and the long-dashed gray lines as a population in which the ammonium sulfate components of the particles are aqueous. A comparison between the data and the dashed lines is the basis of the $\operatorname{DRH}(\varepsilon)$ points shown in Fig. 4 (cf. main text for additional explanation). (b) Probability density functions $p(\varepsilon ; T)$ for the different particle types that constitute the four particle sub-populations. Cartoons A through D represent these four particle types, for which organic material is in green and sulfate is in red. The background shading represents the dissolved fraction of ammonium sulfate, ranging from zero in white to unity in black (cf. Eq. A3). The ordinate of $p(\varepsilon ; T)$ for each particle type is scaled to the fractional contribution to the total $p(\varepsilon)$ (i.e., to the number concentration of the particle sub-population). Panels (c) and (d) are as described for (a) and (b) but for efflorescence measurements. Other differences, especially as related to the gray squares, are described in the main text. For all panels, $d_{\mathrm{m},+1}^{\mathrm{mono}}=90 \mathrm{~nm}$.

$\varepsilon>0.8$. Case $a$ seems untenable because the ERH of the mixed material should not, under most circumstances, be greater than that of the pure material (30-35\%), though cooperative nucleation cannot be ruled out beyond possibility. The more expected phenomenon is a mix of cases $b$ and $c$, with case $b$ holding true for $\varepsilon \rightarrow 0.8$ and case $c$ for $\varepsilon \rightarrow 1$.

\subsubsection{Number-diameter distributions}

The number-diameter distribution measurements were used to probe the deliquescence transitions of the particle popula- tion for DRH $<60 \%$, thereby extending the transmission ratio measurements. Data were also collected for DRH $>60 \%$ to test for confirmation of the results obtained by the transmission ratio measurements.

The hygroscopic response of the number-diameter distribution of a particle population to changes in $\mathrm{RH}$ depends on the organic volume fraction distribution $p(\varepsilon)$ of that population. For our analysis, the hygroscopic response is modeled (described in detail below), and the model result is compared to the measurement. In this analysis, the value $\varepsilon$ above which ammonium sulfate fully dissolves (i.e., final 
deliquescence) is optimized until the model result converges with the measurement. This optimized value of $\varepsilon$ for final deliquescence is associated with the $\mathrm{RH}$ value of the measurement.

Figure 3a shows an example of $N(d)$ at $52 \% \mathrm{RH}$, meaning $\delta=45 \%, x_{a}=52 \%$ (gray squares), $x_{b}=90 \%$ (black squares), and $y=52 \%$ in the following protocols:

$$
\begin{aligned}
40 \% & \rightarrow 7 \% \rightarrow d_{\mathrm{m},+1}^{\mathrm{mono}} \rightarrow x_{a}: 7 \%+\delta \\
& \rightarrow y: 7 \%+\delta \rightarrow N(d, y) \text { (gray squares) }
\end{aligned}
$$

$$
\begin{aligned}
40 \% & \rightarrow 7 \% \rightarrow d_{\mathrm{m},+1}^{\mathrm{mono}} \rightarrow x_{b}: 90 \% \\
& \rightarrow y: 7 \%+\delta \rightarrow N(d, y) \quad \text { (black squares) }
\end{aligned}
$$

for which $x_{a / b}$ represents $\mathrm{RH}_{1}^{\text {nafion }}$ and $y$ represents $\mathrm{RH}_{2}^{\text {nafion }}$. The size distribution represented by the gray squares (Eq. 3a) corresponds to a population in which the ammonium sulfate components of some fraction of the particles are fully deliquesced (i.e., those particles of $\varepsilon>\varepsilon_{\mathrm{D}}(52 \%)$ ). The size distribution represented by the black squares (Eq. 3b) corresponds to a population in which the ammonium sulfate components of all particles are fully deliquesced because of exposure to $90 \% \mathrm{RH}$ and the absence of efflorescence at $52 \% \mathrm{RH}$. A series of experiments of the type represented by Fig. 3a was carried out by scanning $\delta$ from $33 \%$ to $73 \%$, corresponding to $40 \%<y<80 \%$.

Shifts between the size distributions plotted as gray or black squares in Fig. 3 indicate that at least some particles are not fully deliquesced (i.e., particles of $\varepsilon<\varepsilon_{\mathrm{D}}(y)$ are present). For a single value of $y$, the specific value of $\varepsilon_{\mathrm{D}}(y)$ can be determined by scanning $\varepsilon$ and observing $N_{3 \mathrm{~b}}(d, y ; \varepsilon)$ compared to $N_{3 \mathrm{a}}(d, y ; \varepsilon)$. This statement is explained by the following line of reasoning. The RH histories of Eqs. (3a) and (3b) begin at $40 \%$. Depending on the overall composition $\varepsilon$ of a particle, the ammonium sulfate component at $40 \% \mathrm{RH}$ in the CMFR outflow is, by definition, either partially solid for $\varepsilon<\varepsilon_{\mathrm{D}}(40 \%)$ or completely dissolved for $\varepsilon \geq \varepsilon_{\mathrm{D}}(40 \%)$. For a particle population characterized by a single organic volume fraction $\varepsilon$, a comparison of $N(d, y ; \varepsilon)$ of Eq. (3a) to that of $N(d, y ; \varepsilon)$ of Eq. (3b) has sixteen possible outcomes to consider based on whether the following conditions are true or false: $\mathrm{DRH}(\varepsilon)>40 \%, \mathrm{ERH}(\varepsilon)>7 \%$, $\operatorname{DRH}(\varepsilon)>y$, and $\operatorname{ERH}(\varepsilon)>y$. Evaluation of these 16 cases, however, shows that there is redundancy among the cases, and a condensed statement can be written as follows (cf. Tables $\mathrm{S} 2$ and $\mathrm{S} 3$ ):

$N_{3 \mathrm{~b}}(d, y ; \varepsilon)$ compared to $N_{3 \mathrm{a}}(d, y ; \varepsilon)$ is

$\begin{cases}\text { shifted right, } & \text { for }\left(\varepsilon<\varepsilon_{\mathrm{D}}(40 \%) \text { and } \operatorname{DRH}(\varepsilon) \geq y\right) \\ \text { identical, } & \text { for }\left(\varepsilon<\varepsilon_{\mathrm{D}}(40 \%) \text { and } \operatorname{DRH}(\varepsilon)<y\right) \\ \text { identical, } & \text { for } \varepsilon \geq \varepsilon_{\mathrm{D}}(40 \%)\end{cases}$

Therefore, in the case that $\varepsilon$ can be scanned at constant $y$ from a low to a high value (i.e., $\left.0<\varepsilon<\varepsilon_{\mathrm{D}}(40 \%)\right), \varepsilon_{\mathrm{D}}(y)$ and hence $\operatorname{DRH}(\varepsilon)$ are obtained by noting at what value of $\varepsilon$ an abrupt change occurs in $N_{3 \mathrm{a}}(d, y ; \varepsilon)$ such that $N_{3 \mathrm{~b}}(d, y ; \varepsilon)$ compared to $N_{3 \mathrm{a}}(d, y ; \varepsilon)$ is changed from right-shifted to identical overlap.

The particle population of Fig. $3 \mathrm{a}$ is characterized by a broad distribution $p(\varepsilon ; T)$ (i.e., Fig. $3 \mathrm{~b}$ ) and thus a range of epsilon contributes to the observations. The black squares compared to the gray squares of Fig. 3a represent the comparison of $N_{3 \mathrm{~b}}(d, y)$ to $N_{3 \mathrm{a}}(d, y)$. The dependency of relative shifts between $N_{3 \mathrm{a}}(d, y ; \varepsilon)$ and $N_{3 \mathrm{~b}}(d, y ; \varepsilon)$ on $\varepsilon$ underlies any comparison between the two data sets of Fig. 3a. The rightshift of $N_{3 \mathrm{~b}}(d, y)$ compared to $N_{3 \mathrm{a}}(d, y)$ indicates that particles characterized by $\varepsilon<\varepsilon_{\mathrm{D}}(40 \%)$ are present in the population. Further interpretation of the shifts by the unaided eye is not possible because their effects are convoluted with the dispersity of $p(\varepsilon ; T)$.

A forward hygroscopic model that incorporates $p(\varepsilon ; T)$ of the four particle types is used to aid in the interpretation of data sets represented in Fig. 3a. The initial numberdiameter distributions used as inputs represent particle types A through D (cf. Sect. 3.1). These initial distributions for each particle type are evolved in the model according to the RH exposures given either by Eq. (3a) or (b), including phase transitions (cf. Fig. S5). The analysis optimizes the parameter $\varepsilon_{\mathrm{D}}(y)$ to minimize the difference between model prediction and observed data. The optimized model predictions (dashed red line in Fig. 3a) compare well to the data (gray squares). The dashed red line is the sum result for the four particle types, and the individual results for the different particle types are shown by the four solid color lines. The result for $\varepsilon_{\mathrm{D}}(52 \%)$ is plotted as a data point of $\mathrm{DRH}(0.82)=52 \%$ in Fig. 4. The results of similar experiments carried out at other values of $y$ are represented as the data points of Fig. 4.

The number-diameter distribution measurements were also used to extend the efflorescence measurements to lower RH values than probed by the transmission ratio measurements (i.e., to ERH values below $15 \%$ ). Two different protocols were used (cf. Sects. 2.2.2 and 2.2.3). For the first protocol, the settings $x_{a / b}: \mathrm{RH}_{1}^{\text {nafion }}$ and $y: \mathrm{RH}_{2}^{\text {nafion }}$ were as follows:

$$
\begin{aligned}
40 \% & \rightarrow 90 \% \rightarrow 7 \% \rightarrow d_{\mathrm{m},+1}^{\mathrm{mono}} \rightarrow x_{a}: 50 \% \\
& \rightarrow y: 50 \% \rightarrow N(d, y) \text { (gray squares) } \\
40 \% & \rightarrow 90 \% \rightarrow 7 \% \rightarrow d_{\mathrm{m},+1}^{\text {mono }} \rightarrow x_{b}: 90 \% \\
& \rightarrow y: 50 \% \rightarrow N(d, y) \text { (black squares) }
\end{aligned}
$$

The RH histories of Eqs. (5a) and (5b) force full deliquescence of all ammonium sulfate components at $90 \% \mathrm{RH}$. At $7 \% \mathrm{RH}$ in the next step of the $\mathrm{RH}$ sequence, the ammonium sulfate component at least partially crystallizes for particles of $\varepsilon \leq \varepsilon_{\mathrm{E}}(7 \%)$ but does not crystallize at all for particles of $\varepsilon>\varepsilon_{\mathrm{E}}(7 \%)$. 
Table 1. Parameterization of $\mathrm{DRH}(\varepsilon)$ and $\operatorname{ERH}(\varepsilon)$ data sets shown in Fig. 4. Coefficients express equations of the form $\operatorname{DRH}(\varepsilon)=\Sigma_{i}$ $c_{i, d} \varepsilon^{i}$ valid for $0 \leq \varepsilon \leq 0.86$ and $\operatorname{ERH}(\varepsilon)=\Sigma_{i} c_{i, e} \varepsilon^{i}$ valid for $0 \leq$ $\varepsilon \leq 0.55$. The parameterization of $\operatorname{DRH}(\varepsilon)$ is for full deliquescence. These equations for $\mathrm{DRH}(\varepsilon)$ and $\mathrm{ERH}(\varepsilon)$ appear as the dashed lines in Fig. 4. The parameterization is accurate compared to the data within $1.5 \% \mathrm{RH}$ for $\mathrm{DRH}(\varepsilon)$ and $4 \%$ for $\operatorname{ERH}(\varepsilon)$.

\begin{tabular}{lrr}
\hline Coefficient & DRH & ERH \\
\hline$c_{0}$ & 80.67 & 28.35 \\
$c_{1}$ & -11.45 & -13.66 \\
$c_{2}$ & 0 & 0 \\
$c_{3}$ & 57.99 & -83.80 \\
$c_{4}$ & -106.80 & 0 \\
\hline
\end{tabular}

Figure 3c shows an example of the number-diameter distributions obtained for the protocols of Eq. (5a) and (b). The black squares represent a population in which the ammonium sulfate components of all particles are fully deliquesced, and the gray squares represent a population that can have two categories of particles. These categories include: (1) particles of the high organic volume fraction that do not effloresce at $7 \% \mathrm{RH}$ and (2) those of low to intermediate $\varepsilon$ that do effloresce at $7 \% \mathrm{RH}$ and are not fully deliquesced by $50 \% \mathrm{RH}$. The DRH and ERH lines of Fig. 4 imply that an additional possible category of particles, those of intermediate $\varepsilon$ that effloresce at $7 \%$ RH but fully deliquesce by $50 \%$ RH, does not exist for the tested particle populations. The rightward shift apparent in Fig. 3c for the black squares compared to the gray squares arises from the sub-population representing the second category. Particles of the first category have identical diameters for Eq. (5a) and (b).

For the hypothetical experiment of a particle population of monodisperse $\varepsilon$, for a series of measurements that scans from high to low $\varepsilon$, a comparison of $N_{3 \mathrm{a}}(d, y ; \varepsilon)$ to $N_{3 \mathrm{~b}}(d, y ; \varepsilon)$ shows identical distributions until $\varepsilon_{\mathrm{E}}(7 \%)$. In the real experiment, the particle population represented in Fig. $3 \mathrm{~d}$ is characterized by a broad distribution $p(\varepsilon ; T)$. The forward hygroscopic model is employed to match the model output (dashed red line) to the data (gray squares) by optimization of the parameter $\varepsilon_{\mathrm{E}}(7 \%)$. Figure $3 \mathrm{c}$ shows that the model distribution compares well to the data. The result for $\varepsilon_{\mathrm{E}}(7 \%)$ is plotted as a data point of $\operatorname{ERH}(0.55)=7 \%$ in Fig. 4 . The clustering of three data points at $7 \% \mathrm{RH}$ represents three data sets that were independently collected and analyzed.

The second protocol used number-diameter distribution measurements to probe efflorescence transitions for greater than $7 \% \mathrm{RH}$. This protocol followed the RH history of Eq. (2), i.e., $90 \% \rightarrow 50 \% \rightarrow d_{\mathrm{m},+1}^{\mathrm{mono}} \rightarrow y: 50 \%-\delta \rightarrow 50 \%$ (Fig. 1, $\beta$ arm of row 2). In comparison to the transmission mode measurements, somewhat higher values of $\delta$ were achieved ( $41 \%$ compared to $35 \%$ ) for number-diameter distribution measurements by allowing much longer equilibration times for the Nafion tube set to $\mathrm{RH}_{1}^{\text {nafion }}$. From the

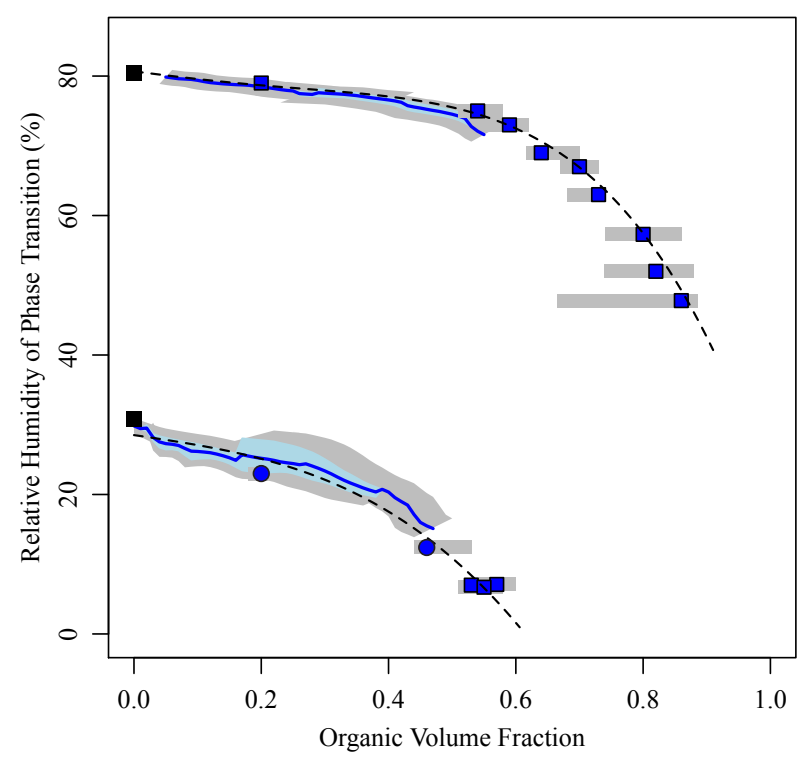

Fig. 4. The deliquescence and efflorescence relative humidities of ammonium sulfate when mixed with isoprene-derived secondary organic material. The solid lines show the $\operatorname{DRH}(\varepsilon)$ and $\operatorname{ERH}(\varepsilon)$ as derived from data sets like panels (a)/(b) and (c)/(d) of Fig. 2. The lines represent the mean across all collected data sets, and the shading around the lines represents the minimum and maximum of the data sets. Squares show $\operatorname{DRH}(\varepsilon)$ and $\operatorname{ERH}(\varepsilon)$ values derived from the type of analysis represented by panels (a)/(b) and (c) $/(\mathbf{d})$ of Fig. 3. Circles show points derived using the second numberdiameter distribution protocol (cf. text). Blue points correspond to isoprene-derived secondary organic material (this study). Black points correspond to pure ammonium sulfate. The dashed lines represent the parameterization equations for $\operatorname{DRH}(\varepsilon)$ and $\operatorname{ERH}(\varepsilon)$ that are given in Table 1 . Gray shaded areas represent the uncertainty in $\operatorname{DRH}(\varepsilon)$ and $\operatorname{ERH}(\varepsilon)$ values, taking into account $\pm 1 \%$ uncertainty in RH and $\pm 1 \mathrm{~nm}$ uncertainty in $d_{\mathrm{m},+1}^{\text {seed }}, d_{\mathrm{m},+1}^{\text {mono }}$, and $d_{\mathrm{m},+1}^{\text {filter }}$ (cf. Tables S4 and S5 for a detailed explanation of the uncertainty calculations).

collected data sets, values $\varepsilon_{\mathrm{E}}(y)$ were again obtained by optimization between the hygroscopic model and the measured data. The results of this protocol appear in Figs. 4 and 5 as the points (circles) for ERH $>7 \%$.

Figure 4 consolidates the data from the transmission ratio experiments as lines and from the number-diameter distribution experiments as points. The two sets of data and analysis methods show a consistent picture of the deliquescence and efflorescence phase transitions of ammonium sulfate mixed with isoprene-derived secondary organic material. Table 1 presents parameterization equations of the $\operatorname{DRH}(\varepsilon)$ and $\operatorname{ERH}(\varepsilon)$ data sets, and these equations are shown as the dashed lines in Fig. 4.

The experiments described thus far were all carried out at $40 \%$ RH in the CMFR with solid ammonium sulfate seed particles. The full matrix of experiments, however, consisted of measurements at $40 \%$ and $60 \% \mathrm{RH}$ and solid and aqueous 


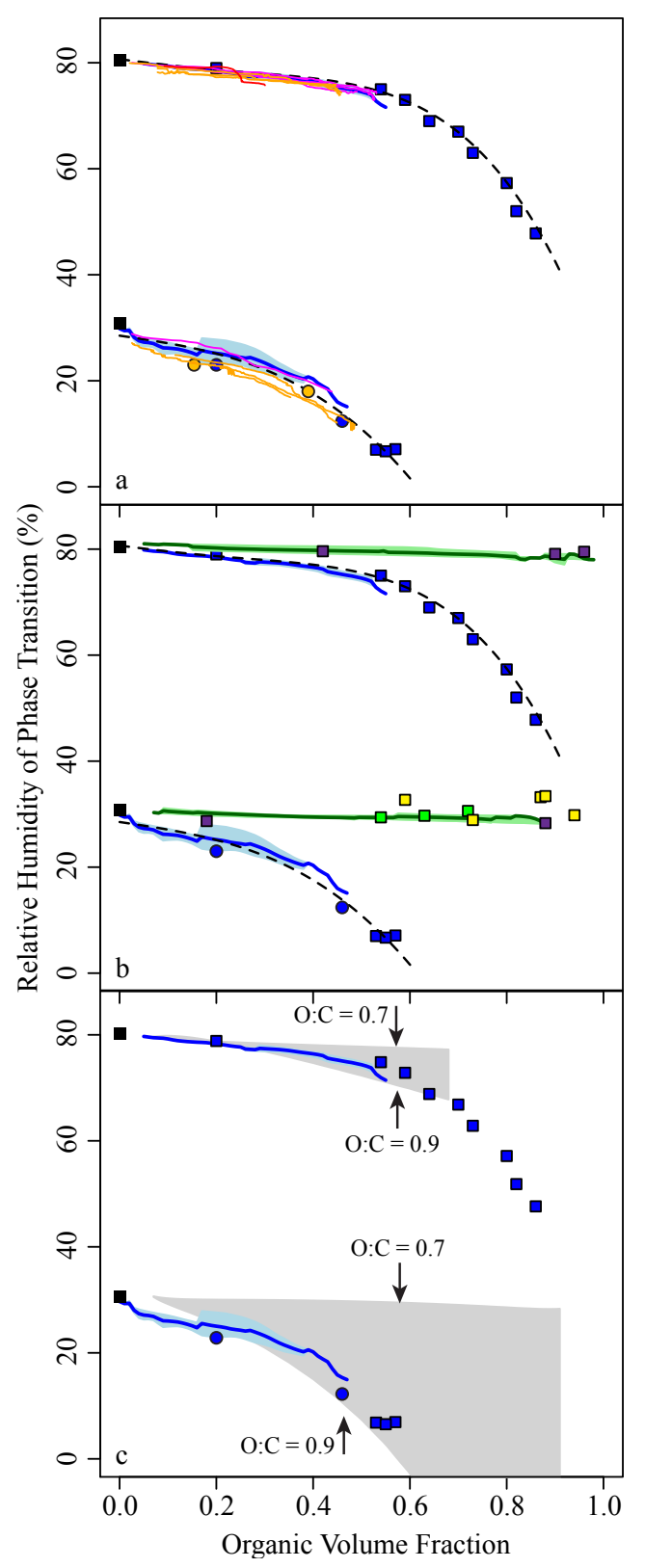

Fig. 5. Additional results and comparison to the literature for the deliquescence and efflorescence relative humidities of ammonium sulfate when mixed with SOM. Blue lines and points reproduce the data set of Fig. 4. (a) Additional results for SOM produced by isoprene photo-oxidation at $60 \% \mathrm{RH}$ with solid sulfate seed particles (magenta), at $40 \% \mathrm{RH}$ with aqueous sulfate seed particles (red), and at $60 \% \mathrm{RH}$ with aqueous sulfate seed particles (orange). (b) Literature results of $\operatorname{DRH}(\varepsilon)$ and $\operatorname{ERH}(\varepsilon)$ for ammonium sulfate mixed with ozonolysis products of $\alpha$-pinene (green shading and lines, Smith et al., 2011; green points, Takahama et al., 2007), limonene (yellow points, Takahama et al., 2007), and $\beta$-caryophyllene (purple points, Bertram et al., 2011). (c) Comparison between the observations of this study and the predictions of Bertram et al. (2011) for $\operatorname{DRH}(\varepsilon)$ and $\operatorname{ERH}(\varepsilon)$ based on O:C ratio (gray shaded area). The upper limit of the gray areas corresponds to the prediction for an $\mathrm{O}: \mathrm{C}$ of 0.7 and the lower limit is the prediction for $\mathrm{O}: \mathrm{C}$ of 0.9 . seed particles, resulting in a total of four conditions. The gas-phase mechanism can be altered by changes in relative humidity (Jonsson et al., 2006). Heterogeneous particlephase chemistry can be altered by particle-phase liquid water content (Jang et al., 2002; Volkamer et al., 2009; Sareen et al., 2010). There can also be increased partitioning of gas phase products to the particle phase (Pankow and Chang, 2008; Barley et al., 2009; Romakkaniemi et al., 2011). Singly or combined, these differences can influence the composition and hence hygroscopic properties of secondary organic material. The results of the conducted experiments, however, show no detectable differences in the phase transitions of the ammonium sulfate for the investigated matrix of four experimental conditions (Fig. 5a). The explanation, with respect to the indifference of the phase transitions, may be tied to the consistent oxygen-to-carbon elemental ratio of the SOM that characterized these four experimental conditions (i.e., 0.67 to $0.74)$.

\subsection{Comparison to other studies}

The results of this study can be compared to other observations of the hygroscopic phase transitions of particles composed of ammonium sulfate and secondary organic material, particularly in relation to the miscibility of the aqueous phases at intermediate relative humidity $(\mathrm{RH}<\mathrm{DRH})$ and the effects thereof on the deliquescence and efflorescence phase transitions of ammonium sulfate. In contrast to the strong dependence of DRH and ERH on $\varepsilon$ that is apparent in Fig. 4 for isoprene-derived SOM, both the DRH and ERH of ammonium sulfate were reduced by no more than $4 \% \mathrm{RH}$ when mixed with SOM produced by $\alpha$-pinene dark ozonolysis products for $0.0<\varepsilon<0.96$ (Takahama et al., 2007; Smith et al., 2011) (Fig. 5b). Similarly, $\beta$-caryophyllene ozonolysis SOM caused at most a $2 \% \mathrm{RH}$ reduction in DRH and ERH for $\varepsilon$ up to 0.96 (Bertram et al., 2011), and limonene ozonolysis SOM caused no statistically different change in the ERH of ammonium sulfate for $\varepsilon$ up to 0.94 (Takahama et al., 2007). In these cases, the explanation for the absence of strong effects, as suggested by Smith et al. (2011), is that separation occurs between the organicwater and inorganic-water phases below some critical relative humidity $(>80 \% \mathrm{RH})$. The phase transitions of ammonium sulfate then proceed approximately as they would for the pure compound. By comparison, the implication of the results shown in Fig. 4 is that the SOM produced by photooxidation of isoprene is phase miscible with aqueous ammonium sulfate, even to low water activity. The smooth decrease of the transmission ratio for the efflorescence experiments are also consistent with full miscibility at the water activities of efflorescence and above (cf. Supplementary Material and Fig. S6).

The difference of phase miscibility in the case of SOM produced by the photo-oxidation of isoprene compared to phase separation for the ozonolyzed terpenes should be 
related to the different compositions of the SOMs and their relative interactions with water and dissolved ammonium sulfate. Although relating SOM physical properties to specific molecules is impractical given the multi-molecular composition, some generalizations may be possible by relating to bulk properties of the SOM. For example, the isoprene SOM presented herein had oxygen-to-carbon $(\mathrm{O}: \mathrm{C})$ ratios ranging from $0.67-0.74( \pm 0.2)$ whereas the $\alpha$-pinene and $\beta$-caryophyllene SOM had lower O:C ratios of $0.39-0.44$ (Smith et al., 2011) and 0.35 (Bertram et al., 2011), respectively. According to the classification system presented in Bertram et al. (2011), the observed phase miscibility and phase separation of ammonium sulfate with these different SOM types are consistent with the differing O:C ratios. The underlying physical phenomenon is the expectation that water-solubility increases for greater O:C ratios, all other factors being equal.

Bertram et al. (2011) present an equation to predict DRH and ERH based on the organic-to-sulfate mass ratio of the particle components and the O:C ratio of the organic material. The comparison of DRH and ERH observations of this study to the values predicted by the equation of Bertram et al. (2011) is shown in Fig. 5c. The predictions are shown as shaded regions that correspond to the measurement uncertainty of the O:C ratio (Aiken et al., 2007). The predictive equation is highly nonlinear in its dependence on $\mathrm{O}: \mathrm{C}$, meaning that the sensitivity of the prediction to the negative side of the uncertainty is very small and that the shaded region in Fig. $5 \mathrm{c}$ represents nearly entirely the positive side of the uncertainty. Figure 5c shows that the DRH observations and predictions are in good agreement across the range of organic volume fractions for which the prediction is valid. Within the uncertainty of the O:C measurement, the ERH observations are also in good agreement with the predictions. The ERH data lie along the lower edge of the shaded region, corresponding to the upper limit of the positive uncertainty of the O:C ratio.

\section{Conclusions and implications}

The results of this study show that secondary organic material produced by isoprene photo-oxidation strongly influences the hygroscopic phase transitions of ammonium sulfate. The SOM was produced at $40 \%$ and $60 \%$ relative humidity, at mass loadings from 20 and $30 \mu \mathrm{g} \mathrm{m}^{-3}$, with O:C ratios from 0.67 and $0.74( \pm 0.2)$, and with aqueous and solid sulfate seed particles. The results are presented in Fig. 4 within the framework of a phase diagram (Berry et al., 2000). The liquidus line is given by $\operatorname{DRH}(\varepsilon)$. This line divides the phase space into a miscible liquid region to the right of the $\operatorname{DRH}(\varepsilon)$ curve and a liquid-solid region to the left of the curve in which a liquid and a solid co-exist (i.e., phase separated).
Compared to a deliquescence relative humidity of $80 \%$ and an efflorescence relative humidity of $30-35 \%$ for pure ammonium sulfate, efflorescence was eliminated for mixed aqueous particles of high organic volume fractions of approximately 0.6 , and the deliquescence relative humidity decreased to $40 \%$ for volume fractions approaching 0.9 . These strong effects caused by isoprene-derived SOM stand in contrast to the minimal influence on ammonium sulfate phase transitions that have been reported for SOM produced by terpene ozonolysis. These different behaviors are rooted in the differing tendencies of the studied SOMs to either mix with or separate from an aqueous inorganic phase. Homogeneous mixing of isoprene-derived SOM with aqueous sulfate lowers both DRH and ERH, increasing for greater organic volume fractions. The decrease in DRH is driven thermodynamically (i.e., the Gibbs-Duhem relation) whereas the decrease in ERH is driven both thermodynamically and kinetically (i.e., the rate of random molecular associations leading to critical germ formation of crystalline ammonium sulfate is decreased by the presence of dissolved organic molecules). Comparison of this study's findings to the results reported in the literature for other types of SOM underscores that secondary organic material composition, which varies with VOC precursor and oxidation conditions, is an important factor governing phase separation and the efflorescence and deliquescence of mixed particles.

These results have several different important implications with respect to atmospheric processes. The hydrolysis of $\mathrm{N}_{2} \mathrm{O}_{5}$ on and in aerosol particles affects the rates of atmospheric ozone production. The availability of water is a key factor influencing the rate of this reaction (Chang et al., 2011). Anttila et al. (2006) observed that the uptake probability of $\mathrm{N}_{2} \mathrm{O}_{5}$ on aqueous ammonium bisulfate particles coated by $\alpha$-pinene ozonolysis products is inversely related to the thickness of the organic coating. The suggestion was that $\mathrm{N}_{2} \mathrm{O}_{5}$ must first dissolve into and then diffuse through the organic layer to reach the aqueous inorganic core and hydrolyze. By comparison, the present study's results show that aqueous ammonium sulfate and isoprene-derived SOM form a mixed phase, ruling out a thick organic coating. Water is expected to be readily available at the surface of the particle and to promote $\mathrm{N}_{2} \mathrm{O}_{5}$ hydrolysis, though this expectation remains to be tested in laboratory conditions. Furthermore, as a result of the decreased DRH and ERH of ammonium sulfate that is caused by the isoprene-derived SOM, the relative humidity conditions for which the particles participate in aqueous-phase reactions such as $\mathrm{N}_{2} \mathrm{O}_{5}$ hydrolysis are significantly extended compared to mixed particles composed of terpene-derived SOM. These differences with SOM type suggest that there are large differences in $\mathrm{N}_{2} \mathrm{O}_{5}$ and $\mathrm{NO}_{3}$ concentrations in urban environments depending on the properties of the local atmospheric SOM (Riemer et al., 2009).

Another important atmospheric process that influences both the atmospheric oxidant cycle as well as the mass concentration of atmospheric particles is the partitioning 
of organic molecules between the gas and particle phases (Chang and Pankow, 2006; Zuend et al., 2010). Particle morphology can change both the thermodynamics and the kinetics of this partitioning. For aqueous ammonium sulfate and terpene-derived SOM, partitioning occurs into two phases, one of which is organic-rich and other of which is inorganic-rich. By comparison, for aqueous ammonium sulfate and isoprene-derived SOM, partitioning occurs into one homogeneously mixed phase of water, ammonium sulfate, and organic material. The thermodynamics and kinetics related to these two morphologies can differ significantly. As an example, for a two-phase particle, a slightly hygroscopic organic-rich phase absorbs some water in response to an increase in $\mathrm{RH}$, leading to at least some increased partitioning of organic molecules from the gas phase. For one-phase particles, however, this phenomenon can be greatly enhanced by the additional water uptake that is associated with the much more hygroscopic dissolved inorganic salt (Zuend et al., 2010). Given the variability in atmospheric particles, such as organic composition, hygroscopicity, and the ratio of organic to inorganic material, systematic studies of how particle water content and the presence of single or multiple phases affect other atmospheric processes are an important area for future study.

\section{Appendix A}

\section{Probability density function $p(\varepsilon)$ of organic volume fraction $\varepsilon$}

\section{A1 CMFR outflow}

The probability density function $p(\varepsilon ; T)$ of organic volume fraction for the different particle types $T$ is modeled using an approach similar to that described previously in Smith et al. (2011), with two updates in the present analysis: (1) the four particle types are individually modeled and (2) the transmission window around $d_{\mathrm{m},+1}^{\text {mono }}$ is taken into account. Sectional number-diameter bins are used to represent the particle population present in the inflow to the CMFR. One group of 23 bins spanning 61-83 $\mathrm{nm}$ physical diameter represents +1 charged particles, and another group of 31 bins spanning 90-120 nm physical diameter represents +2 charge particles. The number concentrations in these bins correspond to the SMPS measurements. The model grows each approximately monodisperse bin of the inflow into a polydisperse numberdiameter distribution in the outflow using the growth equations originally presented in Seinfeld and Pankow (2003) and adapted in Smith et al. (2011). The growth rates are optimized such that the sum of the modeled distributions is in close agreement with the observed distributions in the CMFR outflow (Smith et al., 2011). For each modeled outflow number-diameter distribution, the model also tracks the corresponding original inflow diameter $d_{\text {in }}$ of that distribu- tion. An organic volume fraction $\varepsilon$ given by $1-\left(d_{\text {in }} / d\right)^{3}$ can be calculated for any $d$ of each outflow distribution, and the probability density function $p(\varepsilon ; T)$ at any $d$ is constructed by simultaneous consideration of the contributions by all distributions.

\section{A2 DMA ${ }^{\text {mono }}$ outflow}

The +1 and +2 particle groups are recharged as they enter the TDMA. There is correspondingly a doubling in the number of tracked number-diameter distributions: the +1 group splits into +1 and +2 subgroups as does the +2 group. These $+1 /+1,+1 /+2,+2 /+1$, and $+2 /+2$ subgroups can be identified as the four particle types $\mathrm{A}, \mathrm{B}, \mathrm{C}$, and D introduced in the main text. The model propagates the number-diameter distributions of the groups representing the four particles types through the transfer function of $\mathrm{DMA}^{\text {mono }}$. A nondiffusing triangular transfer function is used (Knutson and Whitby, 1975). Each post-DMA distribution is still tracked to the original corresponding inflow diameter $d_{\text {in }}$ so that the organic volume fraction $\varepsilon$ is calculable at any $d$ for each distribution. The number concentration $n(\varepsilon ; T)$ that is associated with any organic volume fraction is calculated for each particle type by taking the appropriate sum across all distributions. Based on $n(\varepsilon ; T)$ the probability density function $p(\varepsilon ; T)$ and the cumulative distribution function $P(\varepsilon ; T)$ are calculated for each particle type. Distributions $p(\varepsilon)$ and $P(\varepsilon)$ for the entire particle population are calculated from the underlying $p(\varepsilon ; T)$ and $P(\varepsilon ; T)$ of the particle sub-populations by taking averages that are weighted by the subtotal concentrations.

\section{A3 Water content}

The foregoing analysis leading to $p(\varepsilon)$ and $P(\varepsilon)$ assumes that both $d_{\text {in }}$ and $d$ are composed only of sulfate and organic material. For the number-diameter distribution experiments of Eqs. (3a), (b), (5a), and (b), the size selection in DMA $^{\text {mono }}$ takes place at $7 \% \mathrm{RH}$, and an approximation of negligible water content is acceptably accurate for our data analysis under these conditions. For the transmission ratio experiments, size selection in $\mathrm{DMA}^{\text {mono }}$ takes place at approximately 50 to $60 \%$ RH (i.e., Eqs. 1 and 2), and the water content is not negligible. The nominal diameter of the DMA setpoint is corrected by the water content to obtain the dry diameter $d$ that is needed for $p(\varepsilon)$ and $P(\varepsilon)$. An iterative optimization approach is used for estimating the dry number-diameter distribution, as follows: (1) a dry numberdiameter distribution is assumed, (2) a model of hygroscopic growth is applied to the dry distribution (described in next section), and (3) the modeled distribution is compared to the distribution that is implied by the transfer function of $\mathrm{DMA}^{\text {mono }}$. These steps are repeated iteratively by refining the assumed dry number-diameter distribution until the modeled distribution converges to the implied distribution. Figure S7 shows examples of the optimized dry and the DMA- 
implied distributions of each particle type in deliquescencemode experiments.

\section{A4 Hygroscopic growth}

The volume growth factor $G$ is defined as the particle volume $V(y ; \varepsilon)$ at a relative humidity $y$ because of water uptake divided by the particle volume $V(0)$ in the absence of water. The hygroscopic model uses a fractional mixing rule of independent water uptake, as follows (Stokes and Robinson, 1966; Virkkula et al., 1999):

$$
\begin{aligned}
G(y ; \varepsilon) & =\frac{V(y ; \varepsilon)}{V(0)}=\frac{(1-f) V_{0}+f G_{0}(y) V_{0}+G_{1}(y) V_{1}}{V_{0}+V_{1}} \\
& =(1-\varepsilon)\left(1+f\left(G_{0}(y)-1\right)\right)+\varepsilon G_{1}(y) \text { using } \varepsilon=V_{1} /\left(V_{0}+V_{1}\right)(\mathrm{A} 1)
\end{aligned}
$$

for a particle having an ammonium sulfate component of volume $V_{0}$ in the absence of water and an organic component of volume $V_{1}$ in the absence of water. In the presence of water, the organic volume grows by a factor $G_{1}(y)$. The sulfate component grows to a volume $(1-f) V_{0}+f G_{0}(y) V_{0}$ for an aqueous sulfate growth factor $G_{0}(y)$. The factor $f$ is the fraction of ammonium sulfate that is dissolved (see below). The conceptual model implied by use of Eq. (A1) is that water is taken up independently by the organic and sulfate components even though these three components can occur mixed together in a single phase. This assumption is accurate for ammonium sulfate mixed with $\alpha$-pinene dark ozonolysis SOM (Virkkula et al., 1999; Cocker et al., 2001; Smith et al., 2011) as well as for mixtures of ammonium sulfate and a variety of single organic compounds (Cruz and Pandis, 2000; Choi and Chan, 2002).

The diameter growth factor $g$, which is the theoretical quantity underlying the presentation of diameter changes in Fig. 3a and c, is defined as the particle diameter at a relative humidity $y$ because of water uptake divided by the particle diameter in the absence of water (Swietlicki et al., 2008). The dependence on particle diameter (i.e., Kelvin effect) is assumed negligible for the diameters of the particles of this study. Particles with and without water are taken as spherical. The equation is as follows:

$$
\begin{aligned}
g(y ; \varepsilon) & =\frac{d(y ; \varepsilon)}{d(0)}=\left[\frac{V(y ; \varepsilon)}{V(0)}\right]^{1 / 3} \\
& =\left[(1-\varepsilon)\left(1+\left(g_{0}^{3}(y)-1\right) f\right)+\varepsilon g_{1}^{3}(y)\right]^{1 / 3}
\end{aligned}
$$

The quantity $g_{0}(y)$ of ammonium sulfate is known (Biskos et al., 2006).

The growth factor $g_{1}$ of pure-phase isoprene-derived secondary organic material is calculated by inversion of Eq. (A2) for $g_{1}$ and using as inputs (1) the measured data sets for hygroscopic growth of mixed particles on the upper branch of the hysteresis curve (i.e., $f=1$ ), (2) the known $g_{0}(y)$, and (3) the mode value of $p(\varepsilon)$ that is modeled for the data set. The results of the inversion analysis using data from $d_{\mathrm{m},+1}^{\mathrm{mono}}=95,145,150$, and $190 \mathrm{~nm}$ are parameterized as a continuous function $g_{1}(y)$ using a three-parameter fit. The curves of $g_{0}(y)$ and $g_{1}(y)$ are presented in Fig. S8.

The parameter $f$, representing the fraction of the ammonium sulfate that dissolves into aqueous form at relative humidity $y$, is used to treat partial deliquescence in the model. The solid phase has a volume $(1-f) V_{0}$, and the liquid phase has a dry volume (i.e., excluding water) of $f V_{0}+V_{1}$. For $\varepsilon \geq \varepsilon_{\mathrm{D}}(y)$, particles are deliquesced and $f$ is unity. For $\varepsilon<\varepsilon_{\mathrm{D}}(y)$, the average composition must satisfy the relation: $\varepsilon=V_{1} /\left(V_{0}+V_{1}\right)$, which re-arranges to $V_{0}=V_{1}((1-\varepsilon) / \varepsilon)$. The liquid phase must satisfy the liquidus relation: $\varepsilon_{\mathrm{D}}=$ $V_{1} /\left(f V_{0}+V_{1}\right)$. Substitution of the result for $V_{0}$ into the equation for $\varepsilon_{\mathrm{D}}$ leads after re-arrangement to the following result:

$f(y ; \varepsilon)= \begin{cases}\frac{\varepsilon\left(1-\varepsilon_{\mathrm{D}}\right)}{\varepsilon_{D}(1-\varepsilon)}, & \text { for } \varepsilon<\varepsilon_{\mathrm{D}}(y) \\ 1, & \text { for } \varepsilon \geq \varepsilon_{\mathrm{D}}(y)\end{cases}$

The optimization between model and data sets, such as represented by the dashed red line constrained to the gray data points of Fig. 3a, relates fundamentally to optimizing $\varepsilon_{\mathrm{D}}$ of Eq. (A3). The optimized value minimizes the residual of the sum of the squares between the modeled and observed size distributions. Figure $S 9$ presents a variational analysis in $\varepsilon_{\mathrm{D}}$ that demonstrates the optimization.

\section{Supplementary material related to this article is available online at: http://www.atmos-chem-phys.net/12/ 9613/2012/acp-12-9613-2012-supplement.pdf.}

Acknowledgements. This material is based upon work supported by the National Science Foundation under Grant No. 0925467. Any opinions, findings, and conclusions or recommendations expressed in this material are those of the authors and do not necessarily reflect the views of the National Science Foundation. MLS is the recipient of a Graduate Research Environmental Fellowship from the Global Change Education Program of the Department of Energy.

Edited by: A. Kiendler-Scharr

\section{References}

Aiken, A. C., DeCarlo, P. F., and Jimenez, J. L.: Elemental analysis of organic species with electron ionization high-resolution mass spectrometry, Anal. Chem., 79, 8350-8358, 2007.

Anttila, T., Kiendler-Scharr, A., Tillmann, R., and Mentel, T. F.: On the reactive uptake of gaseous compounds by organic-coated aqueous aerosols: Theoretical analysis and application to the heterogeneous hydrolysis of $\mathrm{N}_{2} \mathrm{O}_{5}$, J. Phys. Chem., 110, 1043510443, 2006. 
Barley, M., Topping, D. O., Jenkin, M. E., and McFiggans, G.: Sensitivities of the absorptive partitioning model of secondary organic aerosol formation to the inclusion of water, Atmos. Chem. Phys., 9, 2919-2932, doi:10.5194/acp-9-2919-2009, 2009.

Berry, R. S., Rice, S. A., and Ross, J.: Physical Chemistry, 2nd edn., Oxford University Press, New York, USA, 2000.

Bertram, A. K., Martin, S. T., Hanna, S. J., Smith, M. L., Bodsworth, A., Chen, Q., Kuwata, M., Liu, A., You, Y., and Zorn, S. R.: Predicting the relative humidities of liquid-liquid phase separation, efflorescence, and deliquescence of mixed particles of ammonium sulfate, organic material, and water using the organic-to-sulfate mass ratio of the particle and the oxygen-tocarbon elemental ratio of the organic component, Atmos. Chem. Phys., 11, 10995-11006, doi:10.5194/acp-11-10995-2011, 2011.

Biskos, G., Paulsen, D., Russell, L. M., Buseck, P. R., and Martin, S. T.: Prompt deliquescence and efflorescence of aerosol nanoparticles, Atmos. Chem. Phys., 6, 4633-4642, doi:10.5194/acp-64633-2006, 2006.

Buzorius, G., Zelenyuk, A., Brechtel, F. and Imre, D.: Simultaneous determination of individual ambient particle size, hygroscopicity and composition, Geophys. Res. Lett., 29, 1974, doi:10.1029/2001 $\mathrm{gl014221,2002.}$

Chan, M. N. and Chan, C. K.: Mass transfer effects on the hygroscopic growth of ammonium sulfate particles with a waterinsoluble coating, Atmos. Environ., 41, 4423-4433, 2007.

Chang, E. I. and Pankow, J. F.: Prediction of activity coefficients in liquid aerosol particles containing organic compounds, dissolved inorganic salts, and water - Part 2: Consideration of phase separation effects by an X-UNIFAC model, Atmos. Environ., 40, 6422-6436, 2006.

Chang, W. L., Bhave, P. V., Brown, S. S., Riemer, N., Stutz, J. and Dabdub, D.: Heterogeneous atmospheric chemistry, ambient measurements, and model calculations of $\mathrm{N}_{2} \mathrm{O}_{5}$ : A review, Aerosol Sci. Tech., 45, 665-695, 2011.

Chen, Q., Liu, Y., Donahue, N. M., Shilling, J. E., and Martin, S. T.: Particle-phase chemistry of secondary organic material: Modeled compared to measured $\mathrm{O}: \mathrm{C}$ and $\mathrm{H}: \mathrm{C}$ elemental ratios provide constraints, Environ. Sci. Technol., 45, 4763-4770, doi:10.1021/es104398s, 2011.

Choi, M. Y. and Chan, C. K.: The effects of organic species on the hygroscopic behaviors of inorganic aerosols, Environ. Sci. Technol., 36, 2422-2428, doi:10.1021/es0113293, 2002.

Ciobanu, V. G., Marcolli, C., Krieger, U. K., Weers, U., and Peter, T.: Liquid-liquid phase separation in mixed organic/inorganic aerosol particles, J. Phys. Chem., 113, 10966-10978, 2009.

Cocker, D. R., Clegg, S. L., Flagan, R. C., and Seinfeld, J. H.: The effect of water on gas-particle partitioning of secondary organic aerosol, Part I: alpha-pinene/ozone system, Atmos. Environ., 35, 6049-6072, 2001.

Cruz, C. N. and Pandis, S. N.: Deliquescence and hygroscopic growth of mixed inorganic-organic atmospheric aerosol, Environ. Sci. Technol., 34, 4313-4319, doi:10.1021/es9907109, 2000.

DeCarlo, P. F., Kimmel, J. R., Trimborn, A., Northway, M. J., Jayne, J. T., Aiken, A. C., Gonin, M., Fuhrer, K., Horvath, T., Docherty, K. S., Worsnop, D. R., and Jimenez, J. L.: Field-deployable, high-resolution, time-of-flight aerosol mass spectrometer, Anal. Chem., 78, 8281-8289, 2006.
Duplissy, J., Gysel, M., Sjogren, S., Meyer, N., Good, N., Kammermann, L., Michaud, V., Weigel, R., Martins dos Santos, S., Gruening, C., Villani, P., Laj, P., Sellegri, K., Metzger, A., McFiggans, G. B., Wehrle, G., Richter, R., Dommen, J., Ristovski, Z., Baltensperger, U., and Weingartner, E.: Intercomparison study of six HTDMAs: results and recommendations, Atmos. Meas. Tech., 2, 363-378, doi:10.5194/amt-2-363-2009, 2009.

Ervens, B., Turpin, B. J., and Weber, R. J.: Secondary organic aerosol formation in cloud droplets and aqueous particles (aqSOA): a review of laboratory, field and model studies, Atmos. Chem. Phys., 11, 11069-11102, doi:10.5194/acp-1111069-2011, 2011.

Hallquist, M., Wenger, J. C., Baltensperger, U., Rudich, Y., Simpson, D., Claeys, M., Dommen, J., Donahue, N. M., George, C., Goldstein, A. H., Hamilton, J. F., Herrmann, H., Hoffmann, T., Iinuma, Y., Jang, M., Jenkin, M. E., Jimenez, J. L., Kiendler-Scharr, A., Maenhaut, W., McFiggans, G., Mentel, Th. F., Monod, A., Prévôt, A. S. H., Seinfeld, J. H., Surratt, J. D., Szmigielski, R., and Wildt, J.: The formation, properties and impact of secondary organic aerosol: current and emerging issues, Atmos. Chem. Phys., 9, 5155-5236, doi:10.5194/acp-9-51552009, 2009.

Hameri, K., Vakeva, M., Hansson, H. C., and Laaksonen, A.: Hygroscopic growth of ultrafine ammonium sulphate aerosol measured using an ultrafine tandem differential mobility analyzer, J. Geophys. Res., 105, 22231-22242, 2000.

Jang, M. S., Czoschke, N. M., Lee, S., and Kamens, R. M.: Heterogeneous atmospheric aerosol production by acid-catalyzed particle-phase reactions, Science, 298, 814-817, 2002.

Jonsson, A. M., Hallquist, M., and Ljungstrom, E.: Impact of humidity on the ozone initiated oxidation of limonene, Delta(3)carene, and alpha-pinene, Environ. Sci. Technol., 40, 188-194, doi:10.1021/es051163w, 2006.

King, S. M., Rosenoern, T., Shilling, J. E., Chen, Q., and Martin, S. T.: Increased cloud activation potential of secondary organic aerosol for atmospheric mass loadings, Atmos. Chem. Phys., 9, 2959-2971, doi:10.5194/acp-9-2959-2009, 2009.

Knutson, E. O. and Whitby, K. T.: Aerosol classification by electric mobility: Apparatus, theory, and applications, J. Aerosol Sci., 6, 443-451, 1975.

Kroll, J. H., Ng, N. L., Murphy, S. M., Flagan, R. C., and Seinfeld, J. H.: Secondary organic aerosol formation from isoprene photooxidation, Environ. Sci. Technol., 40, 1869-1877, doi:10.1021/es0524301, 2006.

Kuwata, M. and Martin, S. T.: Particle size distributions following condensational growth in continuous flow aerosol reactors as derived from residence time distributions: Theoretical development and application to secondary organic aerosol, Aerosol Sci. Tech., 46, 937-949, 2012.

Marcolli, C. and Krieger, U. K.: Phase changes during hygroscopic cycles of mixed organic/inorganic model systems of tropospheric aerosols, J. Phys. Chem., 110, 1881-1893, 2006.

Martin, S. T.: Phase transitions of aqueous atmospheric particles, Chem. Rev., 100, 3403-3453, 2000.

Martin, S. T., Rosenoern, T., Chen, Q. and Collins, D. R.: Phase changes of ambient particles in the Southern Great Plains of Oklahoma, Geophys. Res. Lett., 35, L22801, doi:10.1029/2008g1035650, 2008. 
Meyer, N. K., Duplissy, J., Gysel, M., Metzger, A., Dommen, J., Weingartner, E., Alfarra, M. R., Prevot, A. S. H., Fletcher, C., Good, N., McFiggans, G., Jonsson, A. M., Hallquist, M., Baltensperger, U., and Ristovski, Z. D.: Analysis of the hygroscopic and volatile properties of ammonium sulphate seeded and unseeded SOA particles, Atmos. Chem. Phys., 9, 721-732, doi:10.5194/acp-9-721-2009, 2009.

Murphy, D. M., Cziczo, D. J., Froyd, K. D., Hudson, P. K., Matthew, B. M., Middlebrook, A. M., Peltier, R. E., Sullivan, A., Thomson, D. S., and Weber, R. J.: Single-particle mass spectrometry of tropospheric aerosol particles, J. Geophys. Res., 111, D23s32, doi:10.1029/2006jd007340, 2006.

Pankow, J. F.: Gas/particle partitioning of neutral and ionizing compounds to single and multi-phase aerosol particles. 1. Unified modeling framework, Atmos. Environ., 37, 3323-3333, 2003.

Pankow, J. F. and Chang, E. I.: Variation in the sensitivity of predicted levels of atmospheric organic particulate matter (OPM), Environ. Sci. Technol., 42, 7321-7329, doi:10.1021/es8003377, 2008.

Parsons, M. T., Riffell, J. L., and Bertram, A. K.: Crystallization of aqueous inorganic-malonic acid particles: Nucleation rates, dependence on size, and dependence on the ammonium-to-sulfate, J. Phys. Chem., 110, 8108-8115, 2006.

Pratt, K. A. and Prather, K. A.: Aircraft measurements of vertical profiles of aerosol mixing states, J. Geophys. Res., 115, D11305, doi:10.1029/2009jd013150, 2010.

Ravishankara, A. R.: Heterogeneous and multiphase chemistry in the troposphere, Science, 276, 1058-1065, 1997.

Reid, J. P., Dennis-Smither, B. J., Kwamena, N.-O. A., Miles, R. E. H., Hanford, K. L., and Homer, C. J.: The morphology of aerosol particles consisting of hydrophobic and hydrophilic phases: Hydrocarbons, alcohols and fatty acids as the hydrophobic component, Phys. Chem. Chem. Phys., 13, 15559-15572, 2011.

Riemer, N., Vogel, H., Vogel, B., Anttila, T., Kiendler-Scharr, A., and Mentel, T. F.: Relative importance of organic coatings for the heterogeneous hydrolysis of $\mathrm{N}_{2} \mathrm{O}_{5}$ during summer in Europe, J. Geophys. Res., 114, D17307, doi:10.1029/2008jd011369, 2009.

Romakkaniemi, S., Kokkola, H., Smith, J. N., Prisle, N. L., Schwier, A. N., McNeill, V. F., and Laaksonen, A.: Partitioning of semivolatile surface-active compounds between bulk, surface and gas phase, Geophys. Res. Lett., 38, L03807, doi:10.1029/2010g1046147, 2011.

Rosenoern, T., Paulsen, D., and Martin, S. T.: The 1-by-3 tandem differential mobility analyzer for measurement of the irreversibility of the hygroscopic growth factor, Aerosol Sci. Tech., 43, 641652, 2009.

Saathoff, H., Naumann, K. H., Schnaiter, M., Schock, W., Mohler, O., Schurath, U., Weingartner, E., Gysel, M., and Baltensperger, U.: Coating of soot and $\left(\mathrm{NH}_{4}\right)_{2} \mathrm{SO}_{4}$ particles by ozonolysis products of alpha-pinene, J. Aerosol Sci., 34, 1297-1321, 2003.

Sareen, N., Schwier, A. N., Shapiro, E. L., Mitroo, D., and McNeill, V. F.: Secondary organic material formed by methylglyoxal in aqueous aerosol mimics, Atmos. Chem. Phys., 10, 997-1016, doi:10.5194/acp-10-997-2010, 2010.

Seinfeld, J. H. and Pandis, S. N.: Atmospheric Chemistry and Physics: From Air Pollution to Climate Change, 2nd, Wiley, New York, 2006.

Seinfeld, J. H. and Pankow, J. F.: Organic atmospheric particulate material, Annu. Rev. Phys. Chem., 54, 121-140, 2003.
Shilling, J. E., Chen, Q., King, S. M., Rosenoern, T., Kroll, J. H., Worsnop, D. R., McKinney, K. A., and Martin, S. T.: Particle mass yield in secondary organic aerosol formed by the dark ozonolysis of a-pinene, Atmos. Chem. Phys., 8, 2073-2088, doi:10.5194/acp-8-2073-2008, 2008.

Smith, M. L., Kuwata, M., and Martin, S. T.: Secondary organic material produced by the dark ozonolysis of alpha-pinene minimally affects the deliquescence and efflorescence of ammonium sulfate, Aerosol Sci. Tech., 45, 244-261, 2011.

Song, M., Marcolli, C., Krieger, U. K., Zuend, A., and Peter, T.: Liquid-liquid phase separation and morphology of internally mixed dicarboxylic acids/ammonium sulfate/water particles, Atmos. Chem. Phys., 12, 2691-2712, doi:10.5194/acp-12-26912012, 2012.

Stokes, R. H. and Robinson, R. A.: Interactions in aqueous nonelectrolyte solutions. 1. Solute-solvent equilibria., J. Phys. Chem., 70, 2126-2131, 1966.

Swietlicki, E., Hansson, H. C., Hämeri, K., Svenningsson, B., Massling, A., McFiggans, G., McMurry, P. H., Petäjä, T., Tunved, P., Gysel, M., Topping, D., Weingartner, E., Baltensperger, U., Rissler, J., Wiedensohler, A., and Kulmala, M.: Hygroscopic properties of submicrometer atmospheric aerosol particles measured with H-TDMA instruments in various environments - a review, Tellus: Series B, 60, 432-469, 2008.

Takahama, S., Pathak, R. K., and Pandis, S. N.: Efflorescence transitions of ammonium sulfate particles coated with secondary organic aerosol, Environ. Sci. Technol., 41, 2289-2295, doi:10.1021/es0619915, 2007.

Tang, I. N. and Munkelwitz, H. R.: Composition and temperaturedependence of the deliquescence properties of hygroscopic aerosols, Atmos. Environ., 27, 467-473, 1993.

Varutbangkul, V., Brechtel, F. J., Bahreini, R., Ng, N. L., Keywood, M. D., Kroll, J. H., Flagan, R. C., Seinfeld, J. H., Lee, A., and Goldstein, A. H.: Hygroscopicity of secondary organic aerosols formed by oxidation of cycloalkenes, monoterpenes, sesquiterpenes, and related compounds, Atmos. Chem. Phys., 6, 23672388, doi:10.5194/acp-6-2367-2006, 2006.

Virkkula, A., Van Dingenen, R., Raes, F., and Hjorth, J.: Hygroscopic properties of aerosol formed by oxidation of limonene, alpha-pinene, and beta-pinene, J. Geophys. Res., 104, 35693579, 1999.

Volkamer, R., Ziemann, P. J., and Molina, M. J.: Secondary Organic Aerosol Formation from Acetylene $\left(\mathrm{C}_{2} \mathrm{H}_{2}\right)$ : seed effect on SOA yields due to organic photochemistry in the aerosol aqueous phase, Atmos. Chem. Phys., 9, 1907-1928, doi:10.5194/acp9-1907-2009, 2009.

Wang, S. C. and Flagan, R. C.: Scanning electrical mobility spectrometer, Aerosol Sci. Tech., 13, 230-240, 1990.

You, Y., Renbaum-Wolff, L., Carreras-Sospedra, M., Hanna, S. J., Hiranuma, N., Kamal, S., Smith, M. L., Zhang, X., Weber, R. J., Shilling, J. E., Dabdub, D., Martin, S. T., and Bertram, A. K.: Images reveal that atmospheric particles can undergo liquidliquid phase separations, P. Natl. Acad. Sci. USA, 109, 1318813193.

Zuend, A., Marcolli, C., Peter, T., and Seinfeld, J. H.: Computation of liquid-liquid equilibria and phase stabilities: implications for RH-dependent gas/particle partitioning of organic-inorganic aerosols, Atmos. Chem. Phys., 10, 7795-7820, doi:10.5194/acp10-7795-2010, 2010. 